\title{
Wie wirksam sperren Sperrklauseln? Die Auswirkung von Prozenthürden auf die Parteienzahl im Bundestag und im internationalen Vergleich
}

\section{Kurzfassung}

Sperrklauseln sperren einerseits Parteien von der parlamentarischen Repräsentation aus, wirken andererseits aber einer Fragmentierung des Parlaments entgegen. In der Abwägung von Vor- und Nachteilen rechtlicher Sperrklauseln hat das Bundesverfassungsgericht in den letzten Jahren die Anwendung von Sperrklauseln bei Kommunal- und Europawahlen verboten. Vor diesem Hintergrund wird am Beispiel des Deutschen Bundestages gefragt, welche Auswirkungen unterschiedliche Prozenthürden auf die Parteienzahl im Bundestag haben könnten. Nach den Ergebnissen einer Simulation zu niedrigeren Sperrklauseln im Zeitraum von 1957 bis 2013 ist der geringste Anstieg der Parteienzahl beim Absenken der Hürde von vier auf drei Prozent und der höchste beim Schritt von zwei auf ein Prozent zu erwarten. Ein internationaler Vergleich des Zusammenhangs der Fragmentierung mit weiteren Variablen legt nahe, dass insbesondere das Alter eines demokratischen Parteiensystems einen deutlichen Einfluss auf die parlamentarische Fragmentierung hat. Die Vorliebe vieler Parteien gerade für die Sperrklausel-Option zur Defragmentierung wird abschließend unter dem Aspekt des „Konkurrenzschutzes“ interpretiert. 


\section{Inhalt}

1. Einleitung 394

2. Sperrklauseln in der politikwissenschaftlichen Debatte 397

3. Das Bundesverfassungsgericht als „Kartellwächter“? 401

4. Nationale Ebene: Wie fragmentiert wäre der Deutsche Bundestag mit niedrigerer Sperrklausel?

5. Internationale Ebene: Lassen sich statistische Zusammenhänge aufzeigen?

6. Fazit: Wie wirksam verhindern Sperrklauseln parlamentarische Fragmentierung?

\section{Einleitung*}

Rechtliche Sperrklauseln werden neben der Wahlkreisgröße als wichtigstes Instrument angesehen, um einen effektiven Ausschluss kleiner und kleinster Parteien von der parlamentarischen Repräsentation zu gewährleisten und einer höheren Parteienfragmentierung entgegenzuwirken. ${ }^{1}$ Diese verbreitete Einschätzung von rechtlichen Sperrklauseln ${ }^{2}$ als geeignete und erforderliche Instrumente zur Verhinderung einer (zusätzlichen) Fragmentierung eines Parteiensystems wurde vom Bundesverfassungsgericht in den letzten Jahren mehrfach hinterfragt. Im Schatten der vom Gericht 2008 angestoßenen Reform zur Beseitigung des negativen Stimmgewichts bei Bundestagswahlen ist im gleichen Jahr noch über einen weiteren Reformbedarf des Wahlrechts in der Bundesrepublik entschieden worden. Dabei ging es um die Unzulässigkeit von Sperrklauseln für die Wahl zu kommunalen Parlamenten in Schleswig-Holstein. ${ }^{3}$ Und am 9. November 2011 erklärte das Gericht mit 5:3 Stimmen auch die Fünf-Prozent-Hürde im Europawahlgesetz, also für die Wahl der

* Für wertvolle Hinweise danke ich Ray Hebestreit, Bettina Westle und den beiden anonymen Gutachtern der Zeitschrift für Politikwissenschaft.

1 Vergleiche Anckar 1997; Bischoff 2009; Klingemann/Wessels 2001; Kramer/Bahr 2012; Nohlen 2009: 520; Taagepera 2002.

2 Zum Unterschied von rechtlichen und theoretischen Sperrklauseln vergleiche Klingemann/Wessels 2001 (283): „In contrast to legal thresholds, theoretical thresholds can account for many other parameters that influence the exclusion or inclusion of parties. District magnitude is one of the most important features." Im Unterschied dazu ergeben sich faktische Hürden (als Mindestanzahl von Stimmen für das Erzielen eine Mandats) aus der Wahlkreisgröße und dem Stimmenverrechungsverfahren.

3 BVerfG, 2 BvK 1/07 vom 13.2.2008, http://www.bverfg.de/entscheidungen/ks20080213_2bvk 000107.html (Stand: 10.7.2013). 
deutschen Abgeordneten zum Europäischen Parlament, für unvereinbar mit den Normen der Wahlrechtsgleichheit und der Chancengleichheit der politischen Parteien. ${ }^{4}$ Daraufhin hatte am 13. Juni 2013 der Deutsche Bundestag mit Zustimmung aller Fraktionen mit Ausnahme der Linken eine Drei-Prozent-Hürde im Europawahlgesetz eingeführt. Nach einer erneuten Klage mehrerer Kleinparteien wurde am 26. Februar 2014 auch diese Regelung vom Gericht als nicht vereinbar mit den Grundsätzen der Wahlrechtsgleichheit und der Chancengleichheit der Parteien verworfen.

Das Verfassungsgericht hat mit seinen Entscheidungen die Frage aufgeworfen, wie notwendig und erforderlich die Sperrklauseln für die Stabilisierungs- bzw. Konzentrationsfunktion des Parteiensystems auf parlamentarischer Ebene gegenwärtig tatsächlich (noch) sind. Die noch bestehenden rechtlichen Fünf-ProzentHürden für die Wahl zum Deutschen Bundestag und zu den Landtagen ${ }^{5}$ sind in der deutschsprachigen Politikwissenschaft relativ wenig diskutierte - und somit wahrscheinlich allgemein akzeptierte - Einrichtungen des politischen Systems.

Allgemein wird davon ausgegangen, dass Sperrklauseln der „Parteienzersplitterung" entgegenwirken, indem die Wähler bereits bei der Stimmabgabe das wahrscheinliche Ergebnis berücksichtigen und bevorzugt ,nützliche Stimmen abgeben, d.h. Parteien wählen, die aller Voraussicht nach die Sperrklausel überspringen können“ (Nohlen 2009: 520). Dieser theoretisch gut begründete psychologische Effekt lässt sich empirisch aber nur schwer einschätzen. Während Stimmen für Kleinparteien offensichtlich keine Chance haben, parlamentarisch wirksam werden zu können außer als fehlende Stimmen für andere Parteien bei der Mehrheitsbildung, gibt es dennoch immer noch einen bestimmten Wähleranteil, der aus Überzeugung solche „wasted votes“ (Anckar 1997) abgibt. Davon zu unterscheiden sind Stimmen für Parteien, die oft nur knapp scheitern und die besser als „Risikostimmen“ beschrieben werden können. Das Bundesverfassungsgericht hat für die kommunale und europäische Ebene entschieden, dass solche ,theoretischen“ Begründungen der

4 https://www.bundesverfassungsgericht.de/pressemitteilungen/bvg11-070.html (Stand: 10.7.2013). Zur Rechtsprechung des Bundesverfassungsgerichts zur Fünf-Prozent-Klausel zwischen 1952 und 1979 siehe Becht 1990: 36 ff. Dort auch der Hinweis, dass die 1979 vom Gericht einstimmig akzeptierte Sperrklausel im Europawahlgesetz unter Aspekten der Verhältnismäßigkeit und Erforderlichkeit problematisch sei: „Eine überzeugende Mehrheitsbildung zu ermöglichen, wird als Hauptaufgabe der Sperrklausel angesehen. Zu Recht wird in der Literatur angefragt, was unter diesem wertenden, konturlosen Kriterium zu verstehen sei angesichts der aufgezeigten, vom Bundesverfassungsgericht auch anerkannten ,Grundzersplitterung im Parlament" (ebd.: 175).

5 Alle Bundesländer wenden eine landesweite Fünf-Prozent-Klausel für die Landtagswahlen an, Bremen getrennt für Bremen und Bremerhaven. Öffnungsklauseln für nationale Minderheiten existieren in Brandenburg (Sorben) und Schleswig-Holstein (dänische Minderheit). Grundmandatsklauseln gibt es in Berlin, Brandenburg, Sachsen und Schleswig-Holstein (Georgii 2010: 8). 
Geeignetheit und Erforderlichkeit von rechtlichen Sperrklauseln zur Gewährleistung der Entscheidungsfähigkeit eines Parlaments nicht ausreichend sind, sondern dass die konkreten, gegenwärtigen Verhältnisse berücksichtigt werden müssen. ${ }^{6}$ Für die kommunale Ebene wird inzwischen in allen Bundesländern auf eine Sperrklausel verzichtet.

Vor dem Hintergrund einer bislang kaum empirisch gestützten Diskussion über Sinn und Zweck der Prozenthürde wird diese Problemlage im Folgenden auf die Frage der Effektivität von nationalen Sperrklauseln zur Vermeidung einer hohen Fragmentierung des Parteiensystems auf parlamentarischer Ebene übertragen. Diese Fragestellung wird in zwei Schritten untersucht: im ersten Schritt am Beispiel des Deutschen Bundestages und im zweiten Schritt in einem internationalen Vergleich. Für die Parteienzahl im Deutschen Bundestag wird in Abschnitt vier gefragt, wie stark sich die Anzahl der Bundestagsparteien bei einer niedrigeren Sperrklausel wahrscheinlich erhöhen würde. Im anschließenden Abschnitt (5) wird untersucht, welche Hinweise es im internationalen Vergleich für die Wirksamkeit von Sperrklauseln zur Begrenzung der Parteienfragmentierung in Parlamenten gibt. Neben der Sperrklausel werden dabei weitere Einflussfaktoren wie die Wahlkreis- und Parlamentsgröße und das Alter des Parteiensystems berücksichtigt. Zweck dieser Abschnitte ist die Erstellung einer empirischen Basis, auf der geklärt werden kann, wie geeignet relativ hohe Sperrklauseln zur Beschränkung der parlamentarischen Parteienfragmentierung sind, die wiederum häufig als Funktionsvoraussetzung parlamentarischer Demokratie genannt wird.

Diese empirische Fragestellung wird in einen theoretischen Erklärungsrahmen der Kartellparteienthese eingebettet (Abschnitt drei und sechs), nach der hohe Sperrklauseln auch die Funktion eines Konkurrenzschutzes durch die größeren bzw. die „Kartellparteien“ haben. Demnach wären (hohe) Sperrklauseln eine relativ attraktive Option für etablierte parlamentarische Parteien, den ,Marktzutritt“ für neue Wettbewerber zu erschweren und die eigenen Stimmenanteile durch Kartellierung des Wählermarktes zu erhöhen.

6 https://www.bundesverfassungsgericht.de/pressemitteilungen/bvg11-070.html (Stand: 10.7.2013). 


\section{Sperrklauseln in der politikwissenschaftlichen Debatte}

Ursachen und Auswirkungen von rechtlichen Sperrklauseln in Wahlsystemen im Allgemeinen sowie im politischen System der Bundesrepublik Deutschland sind bislang kaum empirisch untersucht. Im „Handbuch Wahlforschung“ (Falter/Schoen 2005) werden Sperrklauseln lediglich an einer Stelle kurz erwähnt. Eine breitere Resonanz findet die Problematik dagegen in der rechtswissenschaftlichen Literatur.

Aus historischer Perspektive hat Jesse die Parteieneinflüsse bei der Entstehung und Entwicklung des Wahlrechts der Bundesrepublik Deutschland rekonstruiert. Bereits die Verhandlungen des Parlamentarischen Rates über die Einführung einer Sperrklausel im Grundgesetz und zwischen den Ministerpräsidenten in dieser Frage sind von parteipolitischen Interessen mitbestimmt gewesen. Für Jesse ließen die Vorgänge um die Einführung der Fünf-Prozent-Klausel ,die Motive der Hauptinitiatoren im Zwielicht erscheinen. Das Ziel, eine regierungsfähige Mehrheit zu erleichtern, stand jedenfalls nicht im Vordergrund“" (1985: 222). Es liegt also nahe, zwei Argumentationsstränge bei der Rechtfertigung von Sperrklauseln zu unterscheiden: zum einen ihre Auswirkungen auf die Parteienzahl im Parlament (und ggf. auf elektoraler Ebene), zum anderen deren mögliche Auswirkung auf Regierungsbildung und Regierungsstabilität. Eine größere Anzahl parlamentarischer Parteien, so die implizite Annahme der Befürworter, führe automatisch zu einer schwierigeren und intransparenteren Organisation parlamentarischer Mehrheiten und wirke sich somit negativ auf die ,Regierungsfähigkeit' aus. Davon zu unterscheiden ist die empirisch zu klärende Frage, ob und in welchem Umfang Sperrklauseln die Parteienzahl tatsächlich reduzieren können, auf die in den Abschnitten vier und fünf näher eingegangen wird.

Der Anteil der aufgrund der Sperrklausel unberücksichtigt gebliebenen Stimmen erreichte 2013 mit 15,7 Prozent einen neuen Höchststand (Tab. 1). Der hohe Wert für 1990 lässt sich teilweise durch das Scheitern der Grünen (West) erklären, der für 2013 durch die Stimmen für FDP und AfD. Die Werte in Tabelle 1 legen eine Einteilung von drei Perioden nahe. Bis 1969 blieb ein relativ hoher Stimmenanteil unwirksam, von 1972 bis 1987 dagegen ein sehr geringer Anteil. Trotz getrennter Wahlgebiete für Ost- und Westdeutschland versechsfachte sich der Anteil der unwirksamen Stimmen $1990 \mathrm{im}$ Vergleich zu 1987 und blieb seither auf einem deutlich höheren Niveau als in den 1970er und 1980er Jahren. 
Thomas Krumm

Tabelle 1: Unberücksichtigt gebliebene Stimmen bei Bundestagswahlen seit 1953

\begin{tabular}{|c|c|c|c|c|}
\hline & $\begin{array}{c}\text { Stimmen } \\
\text { insgesamt }\end{array}$ & $\begin{array}{c}\text { Mittelwert } \\
\text { Periode }\end{array}$ & $\begin{array}{l}\text { Anteil an den gültigen } \\
\text { Stimmen }(\%)\end{array}$ & $\begin{array}{c}\text { Mittelwert } \\
\text { Periode (\%) }\end{array}$ \\
\hline 1953 & 2.960 .817 & \multirow{5}{*}{2.173 .029} & 10,74 & \multirow{5}{*}{7,18} \\
\hline 1957 & 3.094 .323 & & 10,34 & \\
\hline 1961 & 1.821 .857 & & 5,77 & \\
\hline 1965 & 1.186 .449 & & 3,63 & \\
\hline 1969 & 1.801 .699 & & 5,46 & \\
\hline 1972 & 351.745 & \multirow{5}{*}{431.249} & 0,93 & \multirow{5}{*}{1,13} \\
\hline 1976 & 333.595 & & 0,88 & \\
\hline 1980 & 753.592 & & 1,98 & \\
\hline 1983 & 204.091 & & 0,52 & \\
\hline 1987 & 513.222 & & 1,35 & \\
\hline 1990 & $3.740 .292^{\mathrm{a}}$ & \multirow{7}{*}{3.290 .634} & 8,05 & \multirow{7}{*}{$\begin{array}{c}7,17 \\
(1990-2009: \\
5,74)\end{array}$} \\
\hline 1994 & 1.698 .766 & & 3,60 & \\
\hline 1998 & 2.899 .820 & & 5,88 & \\
\hline 2002 & $3.376 .001^{\mathrm{b}}$ & & 7,03 & \\
\hline 2005 & 1.857 .610 & & 3,92 & \\
\hline 2009 & 2.606 .902 & & 6,01 & \\
\hline 2013 & $6.855 .044^{\mathrm{c}}$ & & 15,72 & \\
\hline Mittelwert & 2.120 .931 & & 5,40 & \\
\hline
\end{tabular}

Quelle: Eigene Berechnung nach www.bundeswahlleiter.de.

Anmerkungen: 1949 wurde die Prozenthürde auf Landesebene angewandt; ${ }^{a}$ einschließlich der Stimmen für die Grünen (West), ${ }^{\mathrm{b}} 2002$ erhielt die PDS 4 Prozent der Zweitstimmen und zog nur mit zwei Direktmandaten in den Bundestag ein, ${ }^{\mathrm{c}}$ davon 2,08 Mio. für FDP und 2,05 Mio. für AfD.

Häufig argumentieren die Befürworter von Prozenthürden mit einer transparenteren und gerechteren Mehrheits- bzw. Regierungsbildung, bei der Kleinparteien als „Zünglein an der Waage“ keinen überproportionalen Einfluss haben sollten. Die Aufgabe von Wahlen liegt aus dieser Perspektive vor allem darin, ,funktions- und handlungsfähige Organe hervorzubringen [...]. Die Gefahr der Stimmen- und Parteienzersplitterung, deren Folge die Handlungsunfähigkeit des Parlaments sei, stelle einen besonderen zwingenden Grund dar, der eine Verstärkung des Funktionsas- 
pektes durch die Sperrklausel gestatte“ (Becht 1990: 65 f.). Der Zusammenhang von Sperrklausel und Funktionsfähigkeit des Parlaments gilt aus dieser Sicht als ,evident sowie gemeinhin anerkannt" (Strohmeier 2013: 160).

Während die Befürworter der Sperrklausel die funktionale Dimension von Wahlen betonen, stellen Kritiker auf den Repräsentationsaspekt und das aus ihm resultierende Gebot der Wahlrechtsgleichheit (Art. 38 Abs. 1 GG) ab (Becht 1990: 69). Auch die willkürliche Wahl des ,cut off points” wird kritisiert: „Why should a party with 5.1 percent of the votes gain seats while a party with 4.9 percent does not?" (Taagepera/Shugart 1989: 37). Für Jesse (2009: 116) ist die Fünf-Prozent-Hürde ein „sinnvoller Kompromiss“" zwischen der Erleichterung der parlamentarischen Mehrheitsbildung und der Repräsentation politischer Vielfalt durch die Verhältniswahl. Gleichwohl plädiert er ,wegen der offenkundigen Ungerechtigkeit“ gegenüber den Wählern (ebd.: 117) für die Einführung einer Nebenstimme, die dann zum Zuge kommen soll, wenn die mit der Hauptstimme gewählte Partei an der Prozenthürde scheitert. ${ }^{7}$

Ein Effekt kleinformatiger Koalitionen ist, dass auch die Anzahl der parteipolitischen „Vetospieler“ (Tsebelis 2002) auf Regierungsebene sinkt. Dabei ist vor allem von Kleinparteien zu erwarten, klientelistisch insbesondere den Nutzen sehr spezieller Interessen auf Kosten des „Gemeinwohls“ zu maximieren. Schließlich wird durch Sperrklauseln auch das Problem extremistischer Kleinparteien adressiert. Allerdings ist die Frage der Erwünschtheit extremistischer und populistischer Kleinparteien im Parlament analytisch zu trennen von der Konzentrationsfunktion der Sperrklausel auf das Parteiensystem bzw. die parlamentarische Ebene. Extremistische Parteien sind Kleinparteien, aber nicht alle Kleinparteien sind auch extremistisch. Sperrklauseln wirken folglich zwar auch gegen extremistische Parteien - aber nicht nur gegen diese. Damit sind sie zwar geeignete, aber nicht unbedingt verhältnismäßige Instrumente in der Auseinandersetzung mit extremistischen Kleinparteien.

Die Sperrklausel wird in der Bundesrepublik des Weiteren durch die Grundmandateklausel (drei Direktmandate) eingeschränkt. Ursprünglich auf ein Direktmandat begrenzt, kann die Fünf-Prozent-Hürde seit 1956 durch drei gewonnene Direktmandate ersetzt werden. Davon hat zuletzt die PDS profitiert, die 1994 mit 4,3 Prozent zwar an der Sperrklausel scheiterte, aber aufgrund von vier gewonnenen Direktmandaten in Berlin dennoch mit 30 Mandaten nach der Listenwahl in den Bun-

7 Diese von Jesse präferierte Variante der Alternative Vote (AV) mit einer oder mehreren Nebenstimmen ist allerdings in Europa unüblich. In Großbritannien scheiterte ihre Einführung (als Alternative zur einfachen Mehrheitswahl) bei einem Referendum am 5. Mai 2011 mit 68,3 Prozent NeinStimmen (vgl. Saalfeld 2012). 
destag einzog. Eine temporäre Öffnung fand für die Bundestagswahl 1990 statt, als die Sperrklausel für die alten und neuen Bundesländer getrennt angewendet wurde (Winkler 2012: 142). Weitere Ausnahmen bestehen nach $\S 6$ Abs. 4 des Bundeswahlgesetzes für Parteien nationaler Minderheiten (z. B. dänische und sorbische Minderheiten).

Die Urteile des Bundesverfassungsgerichts zwischen 1950 und 1979 zur Sperrklausel hat Becht (1990) in einer juristischen Dissertation aufgearbeitet. In der Reihe „Aktueller Begriff“ der Wissenschaftlichen Dienste des Bundestages diskutiert Arndt (2011) drei Varianten von Sperrklauseln zur Europawahl. Die erste Variante ist das bestehende System von nationalen Sperrklauseln in 14 Mitgliedstaaten für die Wahl zum Europäischen Parlament (EP). Bei einer zweiten Variante würden europaweite Sperrklauseln für europäische Parteienverbünde eingeführt, nach der europäische Parteien zum Beispiel in mindestens einem Viertel der Mitgliedstaaten drei Prozent erreichen müssten, um bei der Mandatszuteilung berücksichtigt zu werden. Die dritte Variante knüpft an die nationalen Sperrklauseln an, fügt aber im europäischen Verbund eine Öffnungsklausel hinzu. Danach könnten auch die Parteien die Sperrklausel überwinden, ,deren Schwesterparteien in den anderen Mitgliedstaaten bereits eine bestimmte Anzahl an Mandaten im EP errungen haben". Diese Variante wird von Arndt als „Europäisierung ohne Vereinheitlichung“ umschrieben; sie könnte politisch leichter Akzeptanz finden als die zweite (ebd.: 2).

Schließlich wird in empirischen Ansätzen gefragt, welchen Einfluss Sperrklauseln in Interaktion mit weiteren Variablen wie der Wahlkreisgröße auf die parlamentarische Fragmentierung haben. Lijphart (1994: 12, 1999: 153) hat im Hinblick auf die parlamentarische Fragmentierung die rechtlichen Sperrklauseln und die Wahlkreisgröße als die zwei Seiten der gleichen Münze bezeichnet. Neben der Wahlkreisgröße sind aber auch Cleavage-Strukturen (Amorim Neto/Cox 1997; Stoll 2008) und soziale Heterogenität (Geys 2006) als Einflussgrößen diskutiert worden. Dabei können institutionelle Faktoren wie die Wahlkreisgröße auch mit ,soziologischen' Faktoren wie Cleavage-Strukturen und Heterogenität kombiniert werden, um die Erklärungskraft der Modelle zu erhöhen, wie Geys (2006) am Beispiel von Belgien gezeigt hat. 


\section{Das Bundesverfassungsgericht als „Kartellwächter“?}

Des Weiteren können Sperrklauseln aus theoretischer Perspektive auch als Ergebnis eines „Parteienkartells“ untersucht werden. Absprachen und die Bildung von Oligopolen bzw. Kartellen ist eine in der Organisationssoziologie bereits von Robert Michels (1989/1911) beobachtete Tendenz zum Selbstschutz bzw. Machterhalt von Eliten in Organisationen. Die Kartellparteienthese knüpft an dieses ,eherne Gesetz der Oligarchie" an, nach dem in Parteiorganisationen ein am Erhalt der Organisation und dem eigenen Nutzen orientiertes Verhalten vorherrscht, dem die inhaltlichen Politikziele oft untergeordnet werden. Auf personeller Ebene bilden sich oligarchische Führungszirkel heraus, für die die Parteiorganisation zunehmend zum Selbstzweck geworden sei. Im Unterschied zu den Grundannahmen der Ökonomischen Theorie der Politik geht die Kartellparteienthese stärker auf einen Teilaspekt rationalen Handelns, die Ausschließungstendenzen moderner Parteien, ein.

Die in den 1990er Jahren entwickelte Kartellparteien-These soll die zunehmende Instrumentalisierung staatlicher Leistungen durch politische Parteien erklären. Parteien werden nach Katz und Mair (1995) als Quasi-Staatsorgane öffentlich subventioniert bzw. erschließen sich immer mehr Ressourcen aus dem Staat anstatt aus der Gesellschaft (oder von ihren Mitgliedern). Die Bindung an die Mitglieder bzw. die soziale Basis nimmt ab und die Professionalisierung parteilicher Aufgaben und Arbeitsabläufe nimmt zu. Vor diesem Hintergrund soll der „Marktzutritt“ neuer Parteien durch Absprachen (Kartellierung) der etablierten Parteien erschwert werden. Dies bezieht sich primär auf den Zugang zu öffentlichen Ressourcen, der für die Nicht-Kartellparteien erschwert werden soll (Katz/Mair 1995), ist jedoch auch auf die Sperrklausel übertragbar. In beiden Fällen wird der politische Wettbewerb zwischen den Parteien durch Absprachen (Kartelle) reduziert. Neuen Parteien wird der Zugang zum „Markt“" erschwert, zugleich wird durch das kartellistische Verhalten auch die Bildung von systemkritischen „Anti-Kartellparteien“ gefördert (ebd.).

Typisch für Oligopole, wird neuen Wettbewerbern der Zugang zum Markt erschwert bzw. werden diese vom bestehenden Kartell „kooptiert“; „,[a]ndererseits vermuten Katz und Mair einen Zusammenhang zwischen der Entstehung von,Kartellparteien' und dem Aufstieg rechtspopulistischer Parteien, welcher als ,AntiKartellparteien-Phänomen“ beschrieben wird" (Helms 2001: 701). Die Entstehung von Anti-System- bzw. Kartellparteien wird durch die Monopolisierung von staatlichen Ressourcen durch die „Mainstreamparteien“, die stärker an der Wahrung eigener Interessen als an der Umsetzung der Interessen der ,einfachen' Bürger interessiert seien, begünstigt (Katz 2011: 228). Solche Herausforderer-Parteien verlangen ein viel stärkeres Commitment von ihren Mitgliedern als die Kartellparteien 
und sie werden stärker durch eine Idee als durch eine soziale Gruppe oder Schicht zusammengehalten: „Parties of this type have also been identified as ,left-libertarian' or ,new right' parties, or as ,movement parties ““ (ebd.).

Aus dieser Sicht gehören die von einer Sperrklausel benachteiligten Kleinparteien zu den Anti-Kartellparteien. Unter den , ausgesperrten' Parteien müsste sich demnach ein hoher Anteil von Merkmalen von ,Anti-Kartellparteien' nachweisen lassen. Nach der Einführung der Drei-Prozent-Hürde im Europawahlgesetz 2013 hatten unter anderem NPD, Graue Panther, Freie Wähler und Piratenpartei (erneut) beim Bundesverfassungsgericht geklagt. Die Linke und einzelne Grünen-Abgeordnete äußerten sich kritisch auch gegenüber der neuen, ,undemokratischen' DreiProzent-Hürde. ${ }^{8}$

Den Kleinparteien stand die „Große Koalition“ aus CDU/CSU, SPD, Bündnis 90/ Die Grünen und FDP gegenüber, mit deren Stimmen im Juni 2013 die neue DreiProzent-Hürde in das Europawahlgesetz aufgenommen wurde. Allerdings muss hinzugefügt werden, dass es Sperrklauseln bereits lange vor der Entstehung von Kartellparteien (nach Katz und Mair) gegeben hat. Die Anwendung der Kartellparteienthese ist in diesem Kontext nicht als alternative Erklärungsweise zur empirischen Vorgehensweise in Abschnitt vier und fünf zu verstehen. Die Erklärung von (hohen) Sperrklauseln durch die Kartellparteienthese setzt bei der ,subjektiven " Sicht der Parteien auf die Sperrklausel an. Ihre Anwendung auf die Sperrklauseln legt nahe, dass die davon profitierenden Parteien von sich aus keine Veränderung des Status quo einleiten werden. Ein solcher Versuch würde bedeuten, dass die Parteien freiwillig auf einen zwar kleinen, aber möglicherweise für die eigene Mehrheit entscheidenden „Marktanteil““ verzichten. Vielmehr ist unter der Bedingung rationalen Handelns (Downs 1968) zu erwarten, dass sich die Kartellparteien nach Möglichkeit auf eine maximal zumutbare Höhe einigen, um eine möglichst hohe „Oligopolistenrente“ auf dem Wählermarkt zu erzielen. Sperrklauseln sind aus dieser Perspektive durch die Marktmacht bestehender „Anbieter“ gesetzte institutionelle Marktzutrittsschranken, die die Kosten für neue Parteien stark erhöhen bzw. die Kosten der parlamentarischen Parteien für das Umstimmen der potenziellen Wähler der Kleinparteien reduzieren.

8 Vergleiche Der Spiegel, 8. Oktober 2013: „Sperrklausel: Piratenpartei klagt gegen Dreiprozenthürde bei Europawahl“", online unter www.spiegel.de/politik/deutschland/sperrklausel-piraten-klagen-gegen-dreiprozenthuerde-bei-europawahl-a-926835.html (Stand: 7.2.2014); Die Zeit, 14. Juni 2013: „Europawahl: NPD klagt gegen Drei-Prozent-Hürde“, online unter: www.zeit.de/politik/deutschland/2013-06/npd-klage-europawahl (Stand: 14.10.2013). Die Fraktion der Linken hat in ihrem Entwurf zur Reform des Bundestagswahlrechts vom Juli 2013 auf eine Fünf-Prozent-Hürde verzichtet (Strohmeier 2013: 157). 
Sperrklauseln sind eine von mehreren rational zu wählenden „vote producing activities“ (Cox 2005: 70) der parlamentarischen Parteien. Um Kosten für „persuasion“ und „mobilization“ im Wahlkampf im Hinblick auf die möglicherweise entscheidenden Wähler der Kleinparteien zu reduzieren, kann es aus dieser Perspektive rational sein, sich alternativ mit anderen parlamentarischen Parteien auf Sperrklauseln zu einigen (,coordination“, vgl. ebd.). Das Argument des Schutzes der Mehrheitsbildung ist aus dieser Perspektive nicht ein Zweck, der durch das Mittel der Sperrklausel erreicht werden soll, sondern Mittel, das dem Zweck der Reduzierung des Wettbewerbs dient.

In dieser Situation kommt dem Bundesverfassungsgericht eine Schlüsselfunktion zu. Bereits bei der Wahlrechtsreform 1956 waren die Parteien durch eine vorherige Entscheidung an eine Obergrenze von fünf Prozent gebunden, die dann durch eine Mischung aus politischer Verhandlungstaktik und juristischen Beschränkungen auch voll ausgeschöpft wurde (Scarrow 2001: 67). Bei der Wahl zum ersten gesamtdeutschen Bundestag 1990 traf das Gericht eine Entscheidung, die die starre Anwendung der Fünf-Prozent-Hürde öffnete. Danach war es möglich, in den Bundestag einzuziehen, auch wenn lediglich auf dem Gebiet der früheren DDR die Sperrklausel überwunden wurde, was der PDS und Bündnis 90 den Einzug in den Bundestag ermöglichte (Klingemann/Wessels 2001: 281).

In einer Entscheidung vom 13. Februar 2008 hatte das Bundesverfassungsgericht die Kriterien für die Zulässigkeit einer Fünf-Prozent-Hürde auf kommunaler Ebene aufgrund einer Klage in Schleswig-Holstein deutlich verschärft. Danach reicht lediglich eine allgemeine Erschwerung der Mehrheitsbildung und Beschlussfassung nicht aus, sondern es muss auch konkret nachgewiesen werden, dass mit hinreichender Wahrscheinlichkeit ,die Funktionsfähigkeit der Volksvertretung ohne Sperrklausel ernsthaft gefährdet wäre“. ${ }^{9}$ Zur Rechtfertigung einer Sperrklausel, die einen erheblichen Eingriff in die Grundsätze der Wahlrechtsgleichheit und die Chancengleichheit darstellt, bedarf es des Nachweises einer erheblichen Störung der parlamentarischen Aufgabenerfüllung und Entscheidungsfunktion durch die zu erwartende höhere Anzahl von Kleinparteien in einem Parlament ohne bzw. mit niedrigerer Sperrklausel. ${ }^{10}$

Beide Einschätzungen, die höhere Parteienzahl und die daraus resultierende Funktionsstörung, müssen durch den Gesetzgeber nicht nur als rein theoretische Möglichkeit begründet, sondern nach den aktuellen Verhältnissen beurteilt werden:

9 BVerfG, 2 BvC 4/10 vom 9.11.2011, Absatz-Nr. 46, http:/www.bverfg.de/entscheidungen/ cs20111109_2bvc000410.html (Stand: 10.7.2013).

10 BVerfG, 2 BvC 4/10 vom 9.11.2011, Absatz-Nr. 92, http://www.bverfg.de/entscheidungen/ cs20111109_2bvc000410.html (Stand: 10.7.2013). 
„Dürfte der Gesetzgeber frei darüber befinden, von welchem Wahrscheinlichkeitsgrad an er Funktionsstörungen in Betracht zieht, würde eine gerichtliche Kontrolle gesetzgeberischer Prognoseentscheidungen, einschließlich deren tatsächlicher Grundlagen, unmöglich gemacht" ${ }^{11}$ Daher ist nach Ansicht des Gerichts die allgemeine und abstrakte Behauptung, dass ohne die Fünf-Prozent-Sperrklausel der Einzug kleinerer Parteien und Wählergemeinschaften in die Parlamente erleichtert und die Willensbildung in diesen Organen erschwert werde, als Eingriff in die Grundsätze der Wahlgleichheit und der Chancengleichheit nicht zu rechtfertigen. ${ }^{12}$

Des Weiteren hat das Bundesverfassungsgericht am 9. November 2011 entschieden, dass der ,mit der Fünf-Prozent-Sperrklausel in § 2 Abs. 7 EuWG verbundene schwerwiegende Eingriff in die Grundsätze der Wahlrechtsgleichheit und Chancengleichheit der politischen Parteien [...] unter den gegebenen rechtlichen und tatsächlichen Verhältnissen nicht zu rechtfertigen" 13 ist. Damit hat sich das Gericht auch von seiner Entscheidung vom Mai 1979 abgesetzt, in der es die im Europawahlgesetz vom 16. Juni 1978 eingeführte Fünf-Prozent-Sperrklausel für die Wahlen zum Europäischen Parlament für zulässig erachtete und dies damit begründet hatte, ,dass die Sperrklausel geeignet und erforderlich sei, um - durch Vermeidung einer übermäßigen Parteienzersplitterung im Parlament - dessen Fähigkeit zu einer überzeugenden Mehrheitsbildung und damit zur Erledigung der dem Europäischen Parlament seinerzeit zugewiesenen Aufgaben zu sichern". 14

Zur siebenten Europawahl vom 7. Juni 2009 sind in Deutschland insgesamt 32 Parteien und politische Vereinigungen angetreten. Die Fünf-Prozent-Hürde überwinden konnten allerdings nur sechs Parteien (CDU, SPD, B'90/Grüne, FDP, Die Linke, CSU). „Auf die sonstigen Parteien und politischen Vereinigungen entfielen insgesamt 10,8\% der gültigen Stimmen. Hiervon hätten ohne die Sperrklausel sieben weitere Parteien und politische Vereinigungen einen Sitz oder zwei Sitze im Europäischen Parlament errungen. "15 Tabelle 2 fasst die tatsächliche sowie die Parteienzahl bei einer niedrigeren Sperrklausel unter der Annahme unveränderten Wahlverhaltens für die Europawahlen 1994 bis 2009 zusammen. Der durchschnittliche Anstieg der Parteienzahl wäre im Kontingent der deutschen MEPs im Zeitraum von 1994 bis 2009 (unter der Annahme unveränderten Wahlverhaltens) beim Ab-

11 Ebd.

12 Ebd.

$13 \mathrm{https} / /$ www.bundesverfassungsgericht.de/pressemitteilungen/bvg11-070.html (Stand: 10.7.2013).

14 BVerfG, 2 BvC 4/10 vom 9.11.2011, Absatz-Nr. 4, http://www.bverfg.de/entscheidungen/ cs20111109_2bvc000410.html (Stand: 10.7.2013).

15 BVerfG, 2 BvC 4/10 vom 9.11.2011, Absatz-Nr. 37 f., http://www.bverfg.de/entscheidungen/ cs20111109_2bvc000410.html (Stand: 10.7.2013). 
senken der Hürde von drei auf zwei Prozent am geringsten und beim Schritt von ein auf null Prozent (völlige Abwesenheit einer Hürde) am höchsten.

Tabelle 2: Parteienzahl deutscher MEPs nach unterschiedlich hohen Sperrklauseln

\begin{tabular}{|c|c|c|c|c|c|c|}
\hline Sperrklausel & $\begin{array}{c}5 \\
\text { Prozent }\end{array}$ & $\begin{array}{c}4 \\
\text { Prozent }\end{array}$ & $\begin{array}{c}3 \\
\text { Prozent }\end{array}$ & $\begin{array}{c}2 \\
\text { Prozent }\end{array}$ & $\begin{array}{c}1 \\
\text { Prozent }\end{array}$ & $\begin{array}{c}0 \\
\text { Prozent }\end{array}$ \\
\hline 2009 & $\begin{array}{c}6 \text { (CDU, SPD, } \\
\text { B`90/Grüne, } \\
\text { FDP, Linke, } \\
\text { CSU) }\end{array}$ & 6 & 6 & 6 & $\begin{array}{c}10(+ \text { Freie } \\
\text { Wähler, REP, } \\
\text { Tierschutz, } \\
\text { FAMILIE) }\end{array}$ & $\begin{array}{l}13 \text { (+ Piraten, } \\
\text { Rentner, ÖDP) }\end{array}$ \\
\hline 2004 & $\begin{array}{c}6 \text { (CDU, SPD, } \\
\text { B`90/Grüne, } \\
\text { CSU, PDS, FDP) }\end{array}$ & 6 & 6 & 6 & $\begin{array}{c}10 \text { (+ REP Tier- } \\
\text { schutz, } \\
\text { GRAUE, } \\
\text { FAMILIE) }\end{array}$ & $\begin{array}{c}14 \text { (+ NPD, ÖDP, } \\
\text { FRAUEN, } \\
\text { Bündnis für } \\
\text { Deutschland) }\end{array}$ \\
\hline 1999 & $\begin{array}{c}5 \text { (CDU, SPD, } \\
\text { CSU, PDS, } \\
\text { B`90/Grüne) }\end{array}$ & 5 & $\begin{array}{c}6 \\
(+\mathrm{FDP})\end{array}$ & 6 & 7 (+ REP) & 8 (+ Tierschutz) \\
\hline 1994 & $\begin{array}{c}4 \text { (SPD, CDU, } \\
\text { B`90/Grüne, } \\
\text { CSU) }\end{array}$ & $\begin{array}{c}6 \text { (+ PDS, } \\
\text { FDP })\end{array}$ & $\begin{array}{c}7 \\
(+\mathrm{REP})\end{array}$ & 7 & $\begin{array}{c}8 \text { (+ Bund } \\
\text { freier Bürger) }\end{array}$ & $\begin{array}{l}12 \text { (+ GRAUE, } \\
\text { ÖDP, Autofahrer, } \\
\text { STATT Partei) }\end{array}$ \\
\hline Mittelwert & 5,25 & 5,75 & 6,25 & 6,25 & 8,75 & 11,75 \\
\hline Differenz & & $+0,5$ & $+0,5$ & 0 & $+2,5$ & +3 \\
\hline
\end{tabular}

Quelle: Nach www.bundeswahlleiter.de, unter der Annahme unveränderten Wahlverhaltens. Für Abkürzungen vergleiche die Anmerkungen zu Tabelle 3.

Bei völliger Abwesenheit einer Sperrklausel 2009 hätten CDU, SPD und B'90/ Grüne jeweils zwei Sitze verloren, FDP und CSU je einen Sitz. Den höchsten Gewinn unter den sieben zusätzlichen Kleinparteien hätten die Freien Wähler mit zwei Mandaten erzielt. Republikaner, Tierschutzpartei, Familien-Partei, Piraten, Rentnerpartei und ÖDP hätten je einen Sitz erlangt. Für die acht Sitze der Linken hätte die Abwesenheit der Sperrklausel keinen Unterschied gemacht (Hipp 2013).

Angesichts der im Europäischen Parlament ohnehin gegebenen Parteienzersplitterung von 162 nationalen Parteien und des relativ hohen Anteils von Kleinparteien sei der mit einer nationalen ,Sperrklausel verbundene Eingriff in die Grundsätze der Wahlrechtsgleichheit und Chancengleichheit der Parteien nicht gerechtfertigt." ${ }^{16}$ Eine solche nationale Hürde sei nicht geeignet, die Zersplitterung des Eu-

16 BVerfG, 2 BvC 4/10 vom 9.11.2011, Absatz-Nr. 37 f., http://www.bverfg.de/entscheidungen/ cs20111109_2bvc000410.html (Stand: 10.7.2013). 
ropäischen Parlaments (EP) zu begrenzen und dessen Arbeitsfähigkeit zu gewährleisten, da das deutsche Abgeordnetenkontingent insgesamt nur 13 Prozent des EP ausmache. Die erfolgreichen Kläger argumentierten, dass ,,bei der Europawahl 2009 nur etwa $1 \%$ der Sitze im Europäischen Parlament von der Sperrklausel betroffen gewesen [sind]. Ohne Sperrklausel wären statt aktuell 162 dann 169 Parteien im Europäischen Parlament vertreten" ${ }^{17}$ Die Sperrklausel im deutschen Europawahlgesetz sei daher nicht geeignet, eine Zersplitterung des Europäischen Parlaments zu verhindern. ${ }^{18}$

Die Verwendung von Sperrklauseln und ihre Vereinbarkeit mit den Prinzipien der Wahlgleichheit und der Chancengleichheit der Parteien kann nach Ansicht des Gerichts „nicht ein für allemal abstrakt beurteilt werden“, sondern muss vielmehr „mit Blick auf ein konkretes Repräsentationsorgan zu einem bestimmten Zeitpunkt gerechtfertigt sein. [...] Eine einmal als zulässig angesehene Sperrklausel darf daher nicht als für alle Zeiten verfassungsrechtlich unbedenklich eingeschätzt werden. Vielmehr kann sich eine abweichende verfassungsrechtliche Beurteilung ergeben, wenn sich die Verhältnisse wesentlich ändern". ${ }^{19}$ Unter Verweis auf diese Rechtsprechung hat das Gericht am 26. Februar 2014 auch die Drei-Prozent-Hürde bei Wahlen zum Europäischen Parlament kassiert. ${ }^{20}$

\section{Nationale Ebene: Wie fragmentiert wäre der Deutsche Bundestag mit nied- rigerer Sperrklausel?}

Um direkte Rückschlüsse von seinen Entscheidungen zur kommunalen Praxis und zum Europawahlgesetz auf die Prozenthürde bei der Wahl zum Deutschen Bundestag zu verhindern, hat das Bundesverfassungsgericht die Notwendigkeit einer Einzelfallprüfung betont. Eine Änderung der Prozenthürde für die Wahl zum Bundestag muss auch die Erwägung berücksichtigen, dass eine solche Wahlrechtsänderung aufgrund der gegebenenfalls zu erwartenden veränderten Mehrheiten im Bundestag dann eventuell nicht mehr rückgängig gemacht werden kann. ${ }^{21}$ Dies ist

17 BVerfG, 2 BvC 4/10 vom 9.11.2011, Absatz-Nr. 41 f., http://www.bverfg.de/entscheidungen/ cs20111109_2bvc000410.html (Stand: 10.7.2013).

18 Ebd.

19 BVerfG, 2 BvC 4/10 vom 9.11.2011, Absatz-Nr. 90, http://www.bverfg.de/entscheidungen/ cs20111109_2bvc000410.html (Stand: 10.7.2013).

$20 \mathrm{http}: / / w w w . \bar{b} u n d e s v e r f a s s u n g s g e r i c h t . d e / e n t s c h e i d u n g e n / e s 20140226 \_2 b v e 000213 . h t m l$ (Stand: 17.3.2014).

21 BVerfG, 2 BvC 4/10 vom 9.11.2011, Absatz-Nr. 94, http://www.bverfg.de/entscheidungen/ cs20111109_2bvc000410.html (Stand: 10.7.2013). 
bei der Wahl zum Europäischen Parlament nicht zu erwarten, da die entsprechenden Regelungen von den nationalen Parlamenten getroffen werden.

Um die Wahrscheinlichkeit einer veränderten Anzahl von politischen Parteien im Bundestag durch eine niedrigere Prozenthürde abschätzen zu können, wurden in Tabelle 3 die wahrscheinliche Anzahl der Parteien im Deutschen Bundestag unter der Annahme unveränderten Wahlverhaltens seit 1949 ermittelt. Da die Annahme eines unveränderten Wahlverhaltens bei einer niedrigeren Prozenthürde aber unwahrscheinlich ist, werden in Tabelle 3 als psychologischer Effekt einer niedrigeren Sperrklausel auch zwei Varianten eines Anstiegs des Stimmenanteils der Kleinparteien bei sinkender Prozenthürde berücksichtigt.

Der Einfachheit halber wird in der folgenden Simulation (vgl. Behnke 2009) von einem linearen Zusammenhang zwischen der Reduzierung der Hürde und der Erhöhung der Unterstützung ausgegangen. $\mathrm{Ob}$ und wie sich das Wahlverhalten zugunsten von Kleinparteien durch ein Absenken der Sperrklausel tatsächlich verändern würde, ist empirisch noch nicht untersucht. Es ist jedoch davon auszugehen, dass bei niedrigeren Hürden der Anreiz für Wähler, sich aus strategischen Gründen gegen eine eigentlich präferierte Kleinpartei zu entscheiden, abnimmt. Nicht berücksichtigt werden kann des Weiteren, ob die möglichen Stimmengewinne für die Kleinparteien durch niedrigere Hürden eher zu Lasten der größeren Parteien gehen oder eher aus dem Reservoir der Nichtwähler stammen würden. Unberücksichtigt bleiben muss auch, wie sich eine einmalige Veränderung der Parteienzahl (z. B. Einzug der NPD 1969) auf zukünftige Wahlen ausgewirkt hätte.

In Tabelle 3 werden drei Simulationen berechnet. In der ersten wird der Anstieg der Parteienzahl durch Absenken der Sperrklausel und gleichbleibender elektoraler Unterstützung für die Kleinparteien berechnet. In der zweiten wird ein Anstieg des Stimmenanteils der Kleinparteien um jeweils zehn Prozent durch eine Absenkung der Sperrklausel um ein Prozent simuliert. Dies heißt beispielsweise für die DreiProzent-Hürde einen Stimmenanstieg um 20 Prozent und für die Zwei-ProzentHürde einen Anstieg um 30 Prozent gegenüber dem faktischen Stimmenanteil bei der Fünf-Prozent-Hürde.

Im dritten Szenario wird ein Anstieg des Stimmenanteils um 20 Prozent für jeden Prozentpunkt simuliert, um den die Sperrklausel abgesenkt wird. Für ein Absenken der Sperrklausel auf zwei Prozent bedeutet dies einen Anstieg der elektoralen Unterstützung der Kleinparteien um 60 Prozent. Die Ein-Prozent-Hürde kommt jeweils der natürlichen Hürde sehr nahe, weshalb auch eine Null-Prozent-Hürde nicht berücksichtigt wird. Berücksichtigt bleibt jeweils die Grundmandatsklausel, die etwa 1994 der PDS mit 4,4 Prozent und 1957 der DP mit nur 3,4 Prozent der Zweitstimmen den Einzug in den Bundestag ermöglicht hat. 
Thomas Krumm

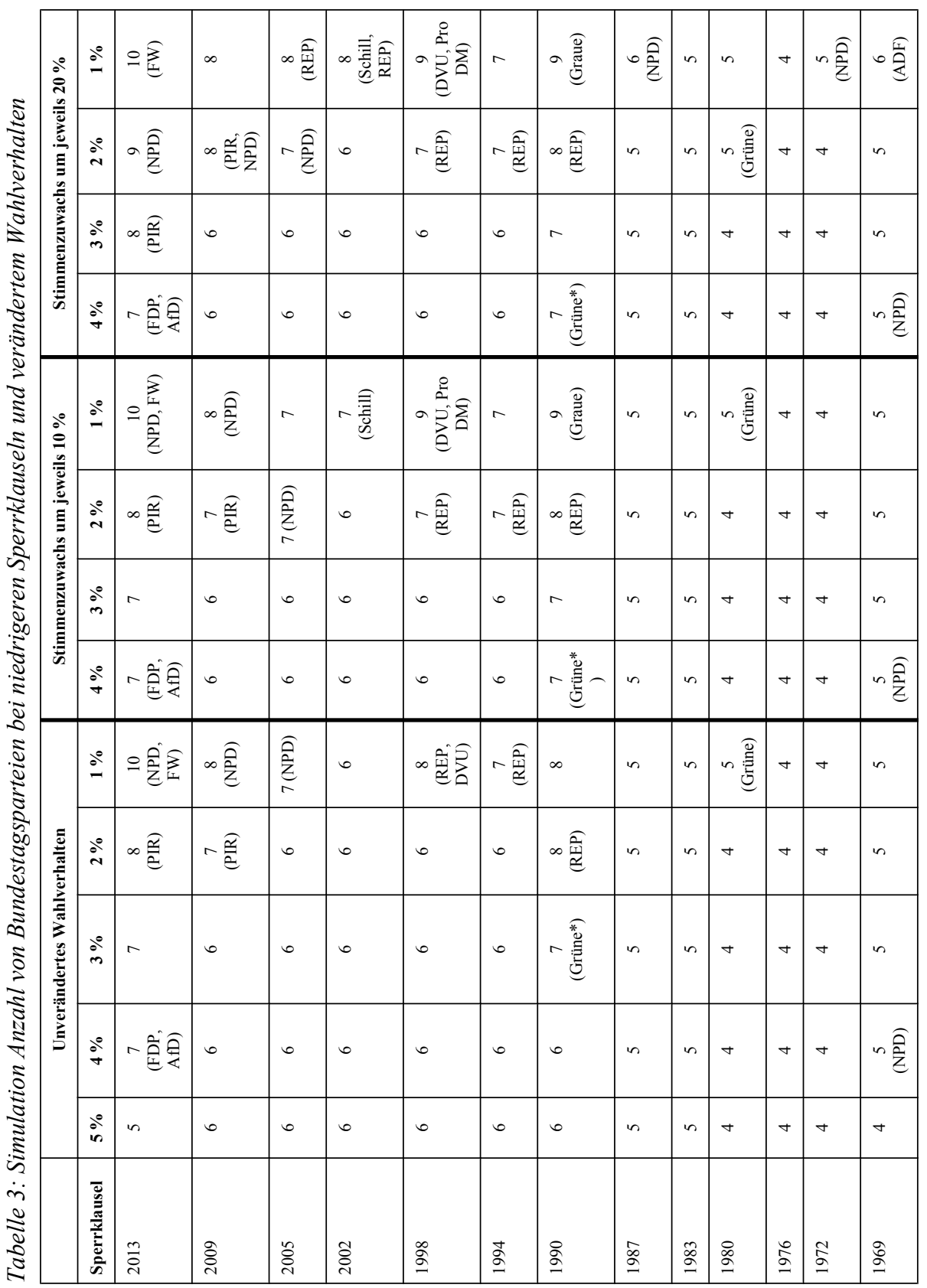


Wie wirksam sperren Sperrklauseln?

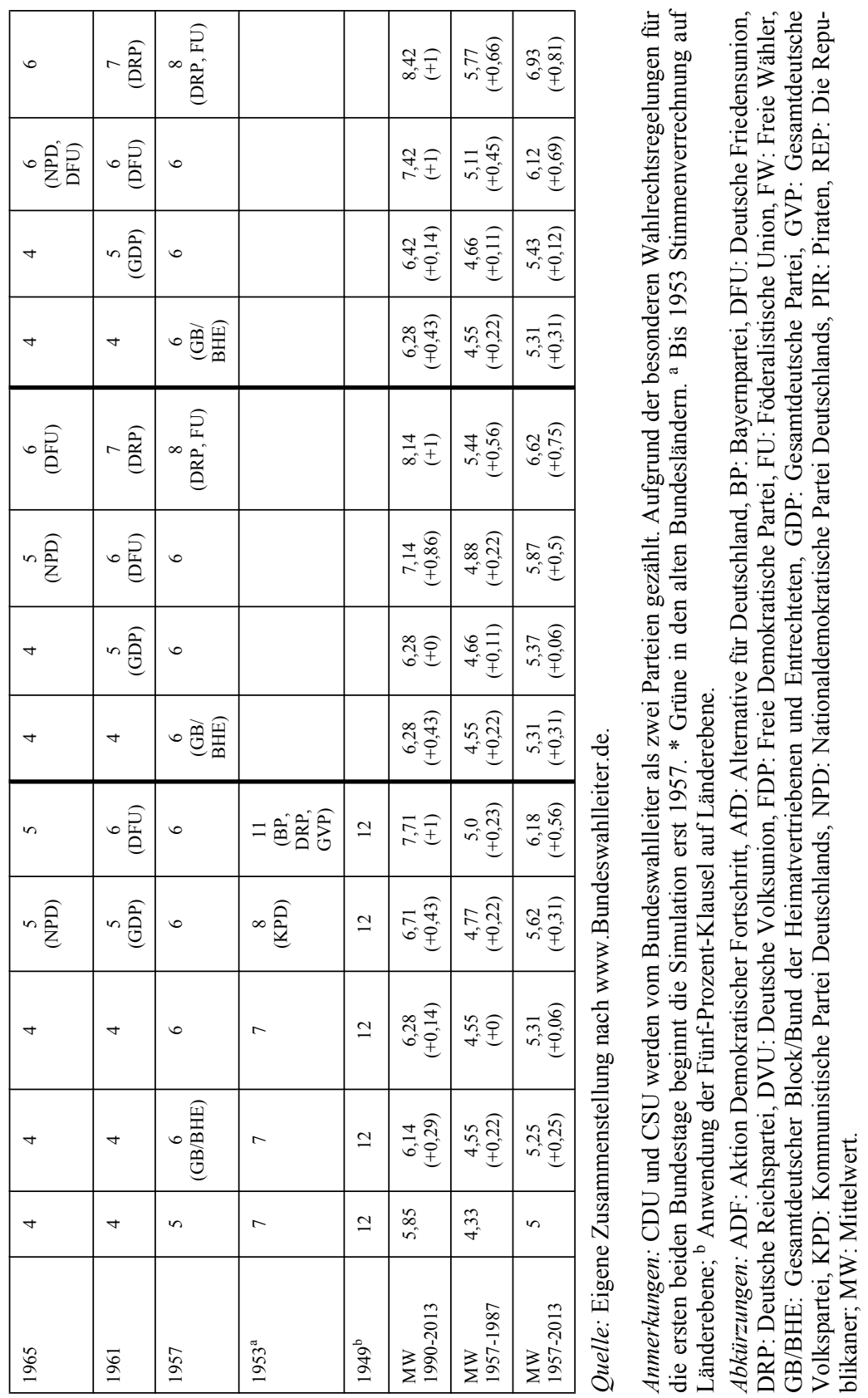


Wie aus Szenario 1 in Tabelle 3 ersichtlich ist, würde auch durch eine erheblich niedrigere Sperrklausel unter der Annahme unveränderten Wahlverhaltens die Anzahl der Parteien im Bundestag nur in geringem Umfang ansteigen. Bei einer VierProzent-Hürde wäre 1957 und 1969 lediglich eine weitere Partei in den Bundestag eingezogen, 2013 allerdings zwei Parteien (FDP, AfD). Bei einer Drei-ProzentHürde und unverändertem Wahlverhalten wären zusätzlich 1990 die Grünen (West) eingezogen und bei einer Zwei-Prozent-Hürde wären 2013 und 2009 die Piraten, 1990 die Republikaner, 1965 die NPD, 1957 die Gesamtdeutsche Partei und 1953 die KPD hinzugekommen. Den größten Anstieg von Parteien im Bundestag hätte es bei einer Ein-Prozent-Hürde 2013 (von 5 auf 10) und 1953 (von 7 auf 11 Parteien) gegeben (wobei 1953 noch die Stimmenverrechnung auf Länderebene zu berücksichtigen ist), 1961 (von 4 auf 6) sowie 1990, 1998 und 2009 (von 6 auf 8). Bei einer Ein-Prozent-Hürde hätte sich - ceteris paribus - jedoch immerhin noch bei fünf Bundestagswahlen gegenüber der aktuellen Hürde nichts geändert. Auffällig ist, dass unter den Kleinparteien, die von einer niedrigen Sperrklausel profitieren würden, häufig rechtsextreme Parteien vertreten sind. Daneben hätten aber auch die Grünen zweimal von einer niedrigeren Hürde profitiert: 1990 hätte ihnen (in den alten Bundesländern) eine Drei-Prozent-Hürde geholfen und 1980 eine Ein-ProzentHürde.

Auch unter der Annahme eines veränderten Wahlverhaltens ergeben sich zunächst nur wenige Neuzugänge im Bundestag. Bei einer Vier-Prozent-Hürde und einem Stimmenanstieg für die Kleinparteien um 10 Prozent wären 2013 wie bereits in Szenario 1 FDP und AfD hinzu gekommen, des Weiteren 1990 (Grüne West), 1969 (NPD) und 1957 (GB/BHE) jeweils eine zusätzliche Partei. Bei einer DreiProzent-Hürde und 20 Prozent Stimmenzuwachs für die Kleinparteien wäre 1961 die GPD hinzugekommen. Bei einer Zwei-Prozent-Hürde (+ 30\%) wäre achtmal eine weitere Partei eingezogen (achtmal unverändert gegenüber der Drei-ProzentHürde). Bei einer Ein-Prozent-Hürde (+ 40 \%) hätte es 2013 mit zehn und 1990 sowie 1998 mit neun die höchste Parteienzahl gegeben (davon 1990 Grüne (West) und B`90/Grüne getrennt gezählt).

Im dritten Szenario wird ein Anstieg des Stimmenanteils der Kleinparteien um 20 Prozent mit jedem Absenken der Sperrklausel um ein Prozent angenommen. Bei einer Vier-Prozent-Hürde (+ 20 \%) wären 2013 FDP und AfD, 1990 die Grünen (West), 1969 die NPD und 1957 der Gesamtdeutsche Block/Bund der Heimatvertriebenen und Entrechteten zusätzlich eingezogen. Bei einer Drei-Prozent-Hürde (+ 40 \%) wären 2013 die Piraten und 1961 die Gesamtdeutsche Partei (GDP) hinzugekommen. Erst bei einer Zwei-Prozent-Hürde und einem Anstieg des Stimmenanteils um 60 Prozent zeigen sich deutlichere Veränderungen. Aber auch unter die- 
sen Annahmen hätte es 2002, 1987, 1983, 1976 und 1972 keine Veränderung gegenüber der aktuellen Fünf-Prozent-Hürde gegeben. Bei einer Ein-Prozent-Hürde und einem Anstieg um 80 Prozent hätte es 1983 und 1976 keine Veränderung gegenüber der Fünf-Prozent-Hürde gegeben. Die stärkste Veränderung hätte es unter diesen Annahmen 1990 (von 6 auf 9), 1961 (von 4 auf 7) und 1957 (von 5 auf 8) gegeben. Allerdings muss für 1990 berücksichtigt werden, dass die Grünen bereits durch Bündnis 90/Die Grünen im Bundestag vertreten waren. Wie in den Jahren 2009, 2005, 2002, 1998, 1969 und 1965 hätte sich die faktische Parteienzahl dann um zwei erhöht. Bemerkenswert ist für alle drei Szenarien eine hohe Stabilität zwischen 1972 und 1987, die auf Ebene der unberücksichtigt gebliebenen Stimmen bereits in Tabelle 1 sichtbar geworden ist. Während der Anstieg 1990 durch die Wiedervereinigung erklärt werden kann, bietet sich als Erklärung für den Wendepunkt 1972 die Polarisierung durch die sozialliberale Koalition an. Im Ergebnis legen die Simulationen einen moderaten durchschnittlichen Anstieg der Parteienzahl nahe. Für die Simulation 1 (unveränderter Stimmenanteil) ergibt sich für den Zeitraum 1957 bis 2013 ein Anstieg der absoluten Parteienzahl um durchschnittlich knapp 0,3 Parteien ${ }^{22}$ durch Absenken der Hürde um je ein Prozent. Dieser durchschnittliche Zuwachs fällt mit + 0,06 Parteien am geringsten aus beim Absenken von vier auf drei Prozent und mit + 0,56 Parteien am höchsten beim Übergang von zwei auf ein Prozent. Für die Perioden 1957-1987 und 1990-2013 findet sich das gleiche Muster.

In Simulation 2 beträgt der durchschnittliche Anstieg 0,4 Parteien ${ }^{23}$ je Prozentpunkt im Zeitraum 1957 bis 2013. Auch in dieser Simulation ist in allen drei Perioden der Anstieg der Parteienzahl beim Absenken von einer Vier- auf eine DreiProzent-Hürde am niedrigsten und beim Übergang von zwei auf ein Prozent am größten. In Simulation 3 beträgt der durchschnittliche Anstieg 0,48 Parteien ${ }^{24}$ je abgesenktem Prozentpunkt für die Gesamtperiode. Wieder ist der Anstieg beim Übergang von vier auf drei Prozent am geringsten (+ 0,12 Parteien) und beim Absenken auf ein Prozent am größten (+ 0,81 Parteien).

In allen drei Simulationen ist der Anstieg beim Absenken der Hürde von vier auf drei Prozent am geringsten und beim Senken von zwei auf ein Prozent am höchsten.

22 Dies entspricht dem Durchschnitt der Veränderung in Szenario 1 über den gesamten Zeitraum bei Absenken von fünf auf ein Prozent (Tabelle 3, letzte Zeile): 6,18 Parteien minus fünf Parteien (Spalte 2) geteilt durch vier ergibt einen durchschnittlichen Zuwachs von 0,295 Parteien je abgesenktem Prozentpunkt der Sperrklausel.

23 Tabelle 3, letzte Zeile: 6,62 Parteien minus fünf Parteien geteilt durch vier ergibt einen Anstieg von durchschnittlich 0,4 Parteien.

24 6,93 Parteien minus fünf Parteien geteilt durch vier ergibt einen durchschnittlichen Zuwachs von 0,48 Parteien. 
Bei der Periodisierung von 1957-1987 und 1990-2013 zeigt sich eine höhere Fragmentierung für die zweite Teilperiode seit 1990. Im Vergleich dieser beiden Perioden kann man also von einem höheren Anstieg der Parteienzahl durch Absenken der Sperrklausel seit der Wiedervereinigung ausgehen, während insbesondere der Zeitraum 1972-1987 durch eine hohe Invarianz gegenüber einer niedrigeren Sperrklausel gekennzeichnet ist.

Da nicht klar ist, zu welchen Anteilen mögliche Stimmenzuwächse der Kleinparteien aus der Gruppe der Nichtwähler bzw. von den parlamentarischen Parteien stammen würden, lassen sich mögliche Verluste für die parlamentarischen Parteien als Folge niedrigerer Sperrklauseln nicht simulieren. Jedoch muss durch einen entsprechenden Abzug von Stimmen von den „Großparteien“ und eine höhere Anzahl parlamentarischer Parteien die Mehrheits- bzw. Regierungsbildung nicht zwangsläufig erschwert werden. So wäre etwa zu berücksichtigen, ob die Stimmen eher von Regierungs- oder Oppositionsparteien abwandern und ob bzw. wie sich eine höhere Parteienzahl im Parlament auf die Koalitionsbildung auswirkt. ${ }^{25}$

\section{Internationale Ebene: Lassen sich statistische Zusammenhänge aufzeigen?}

Um die Effekte von Sperrklauseln auf die Parteienzahl weiter überprüfen zu können, bietet sich ein internationaler Vergleich von Verhältniswahlsystemen mit Sperrklauseln auf nationaler Ebene sowie ohne Sperrklauseln an (für Europa: Nohlen/ Stöver 2010). Ein solcher Effekt könnte sich auf die Anzahl parlamentarischer Parteien (effective number of parliamentary parties, ENPP), aber auch auf die Anzahl elektoraler Parteien (effective number of electoral parties, ENEP; vgl. Laakso/Taagepera 1979) auswirken. Zu erwarten wäre, dass der Effekt auf die Anzahl parlamentarischer Parteien größer ist als der auf die Anzahl elektoraler Parteien. Letzteres würde stärker den psychologischen „Abschreckungseffekt" von Sperrklauseln widerspiegeln, ersteres deren institutionellen Effekt (psychologische und mechanische Dimension). In der folgenden multivariaten Analyse wird zunächst die parlamen-

25 Nach Riker (1962) besteht bei Parteien eine Tendenz zu „minimum winning coalitions“, da bei diesem Koalitionstyp die „Auszahlung“ für die einzelnen Parteien am größten ist. Bei der gegebenen Fünf-Prozent-Hürde ist die minimum winning coalition 2013 eine (nicht realisierte) Koalition aus SPD, Grünen und Linken mit 320 (von 631) Sitzen bzw. 42,7 Prozent der Zweitstimmen. Da über die genaue Sitzverteilung bei simulierten Änderungen der Sperrklausel keine Aussagen möglich sind, müsste eine Schätzung auf Basis der Stimmenanteile erfolgen. Neben der faktischen minimum winning coalition (Rot-Rot-Grün) gäbe es bei einer Vier-Prozent-Hürde (und unverändertem Wahlverhalten) jedoch einige weitere Kandidaten, darunter auch die Optionen CDU/CSU und FDP (46,3\%) sowie CDU/CSU und AfD (46,2\% der Stimmen). Welche dieser Optionen dann die Bedingung einer minimum winning coalition erfüllt und welche der Optionen tatsächlich realisiert würde, hängt nicht zuletzt von der konkreten Umrechung der Stimmenanteile in Sitze ab. 
tarische Fragmentierung, dann die elektorale Fragmentierung als abhängige Variable ausgewählt.

Neben der Sperrklauselhöhe könnte sich auch die Wahlkreisgröße (district magnitude) auf die Fragmentierung auswirken: „Low magnitudes have the same effect as high thresholds: both limit proportionality and the opportunities for small parties to win seats" (Lijphart 1994: 12). Operationalisiert wird diese Variable als durchschnittliche Wahlkreisgröße, das heißt durch eine Division der Anzahl der Sitze der Parlamentskammer durch die Anzahl der Wahlkreise (ebd.: 11). ${ }^{26}$ Da es in einigen Fällen nur einen nationalen Wahlkreis für die relevante Verrechnungsebene gibt, ist die Verteilung der Wahlkreisgröße rechtsschief. Für die Verwendung im linearen Modell wird die Variable daher logarithmiert.

Des Weiteren könnte auch die Größe des Parlaments (operationalisiert als Gesamtzahl der Sitze) Einfluss auf die parlamentarische Fragmentierung haben. $\mathrm{Zu}$ erwarten wäre, dass in größeren Parlamenten auch eine höhere Fragmentierung anzutreffen ist, da bei großen Parlamenten kleinere Parteien bessere Chancen auf Repräsentation haben. Dabei korreliert die Parlamentsgröße im vorliegenden Datensatz nicht mit der durchschnittlichen Wahlkreisgröße; beide Variablen messen also unterschiedliche Aspekte parlamentarischer Repräsentation.

Zudem könnten sich Parteiensysteme mit Verhältniswahlrecht allein durch die Zeit weiter fragmentieren. Daher wird auch der Zeitaspekt mit zwei Variablen getestet: dem demokratischen Alter und einer Wahljahrvariable. Ersterer liegt die Annahme zugrunde, dass sich Parteiensysteme in älteren Demokratien insbesondere unter Bedingungen der Verhältniswahl im Laufe der Zeit weiter aufspalten können. Im Unterschied zur Mehrheitswahl sind die Opportunitätskosten der Abspaltung von einer bestehenden Partei im Fall innerparteilicher Konflikte bei Proporz- deutlich niedriger als bei Majorzverfahren. Dies lässt sich am Parteiensystem der Schweiz gut illustrieren, in dem eine Reihe von Parteien im Laufe der Zeit als Abspaltungen von älteren Parteien entstanden sind (z. B. Bürgerlich-Demokratische Partei, Grünliberale, Schweizerische Volkspartei; Krumm 2013: 200).

Schließlich wird als weitere Variable berücksichtigt, ob das Querschnittsjahr 2010 im jeweiligen Land ein Wahljahr gewesen ist (Dummy-Variable). Durch Parlamentswahlen und die damit verbundene Polarisierung könnte sich die Parteienzahl erhöht haben. War 2010 kein Wahljahr, dann geben die Werte der abhängigen Variablen (ENEP und ENPP) die Fragmentierung der letzten Wahl vor 2010 wieder,

26 Alternativ zu den Variablen Sperrklausel und Wahlkreisgröße könnten auch die „effektiv thresholds" nach Lijphart (1994) berücksichtigt werden, die als kombiniertes Maß für die Fragestellung jedoch weniger geeignet erscheinen. 
für beispielsweise Deutschland von 2009, für die Schweiz von 2007. In diesen Fällen erhält der Variablenwert eine Null. War 2010 dagegen ein Wahljahr, geben die beiden abhängigen Variablen die 2010 durch Wahlen aktualisierten Werte wieder und die Wahljahr-Variable wurde mit einer Eins codiert. Steigt die Fragmentierung tatsächlich mit der Zeit, müssten die mit Eins codierten Länder eine höhere Fragmentierung haben als jene, in denen die Fragmentierungswerte aus einer früheren Wahl stammen (z. B. aus 2007 für die Schweiz). Die Variable Wahljahr prüft also, ob der Effekt einer Fragmentierung durch die Zeit auch kleinschrittig beim Übergang von der vorhergehenden zur nächsten Wahlperiode (2010) zu beobachten ist. Da aber keine Längsschnittdaten verwendet werden, wird lediglich der Zeitraum um 2010 im Datensatz abgebildet.

Nicht berücksichtigt werden kann im gegebenen Rahmen die soziale Heterogenität (Clark/Golder 2006) sowie Details zu den Wahlsystemen (z. B. die Anzahl der Verrechnungsebenen). Auch Sonderregelungen zu Grundmandatsklauseln (Deutschland, Österreich) oder für Parteienbündnisse (in osteuropäischen Staaten) bleiben unberücksichtigt. Sowohl die Nichtberücksichtigung sozioökonomischer Variablen wie auch solcher zu den Details der Stimmenverrechung legen eine vorsichtige Interpretation der Regressionsergebnisse nahe.

In Europa werden hohe Sperrklauseln auf nationaler Ebene relativ häufig in osteuropäischen Ländern einschließlich Russland (seit 2005) angewendet (Nohlen 2009: 185), während die westlichen Nachbarn mit Verhältniswahlrecht teilweise darauf verzichten oder niedrigere Hürden eingerichtet haben. So gilt zum Beispiel in Polen eine Fünf-Prozent-Hürde, in Österreich sind es vier Prozent, in der Schweiz null Prozent. Die Fallauswahl wird durch die Datenlage mitbestimmt. Die Daten zu den abhängigen Variablen (ENPP, ENEP) wurden aus Gallagher (2013) entnommen, die Daten zu den unabhängigen Variablen aus der Databank of Political Institutions (DPI) der Weltbank (Keefer 2012) und nach Abgleich mit Nohlen (2009) und eigenen Recherchen ergänzt. Tabelle 4 fasst die Querschnittsdaten zusammen.

Zunächst werden die möglichen Effekte auf die ENPP in einer multiplen linearen $^{27}$ Regression in mehreren Modellen getestet (Tab. 5). In Modell 1 werden zu-

27 Um eine mögliche Kurvilinearität des Zusammenhangs von ENPP und Sperrklausel besser abschätzten zu können, wurde unter anderem auch eine quadrierte Sperrklausel getestet. Die $\mathrm{R}^{2}$-Werte konnten dadurch jedoch nicht verbessert werden. Daher wird im Folgenden die einfache Sperrklausel weiter verwendet. 
Wie wirksam sperren Sperrklauseln?

Tabelle 4: Übersicht Fälle und Variablen im internationalen Vergleich (Jahr 2010)

\begin{tabular}{|c|c|c|c|c|c|c|c|}
\hline & ENPP & ENEP & $\begin{array}{l}\text { Höhe der } \\
\text { Sperrklausel }\end{array}$ & $\begin{array}{l}\text { MW Wahl- } \\
\text { kreis }\end{array}$ & $\begin{array}{l}\text { Parlaments- } \\
\text { sitze }\end{array}$ & $\begin{array}{l}\text { Alter des } \\
\text { Parteien- } \\
\text { systems }\end{array}$ & $\begin{array}{l}\text { Wahl- } \\
\text { jahr }\end{array}$ \\
\hline Brasilien & 10,44 & 11,26 & 5 & 19 & 513 & 21 & 1 \\
\hline Bulgarien & 3,34 & 4,4 & 4 & 7,7 & 240 & 20 & 0 \\
\hline Dänemark & 5,33 & 5,41 & 2 & 10,5 & 179 & 80 & 0 \\
\hline Deutschland & 4,83 & 5,58 & 5 & 598 & 622 & 62 & 0 \\
\hline $\begin{array}{l}\text { Dominikanische } \\
\text { Republik }\end{array}$ & 2,01 & 2,91 & 0 & 6,1 & 183 & 14 & 1 \\
\hline El Salvador & 2,94 & 2,92 & 0 & 5,6 & 84 & 26 & 0 \\
\hline Estland & 4,37 & 5,02 & 5 & 8,4 & 101 & 18 & 0 \\
\hline Finnland & 5,13 & 5,88 & 0 & 13,3 & 200 & 80 & 0 \\
\hline Georgien* & 1,55 & 2,58 & 5 & 77 & 150 & 6 & 0 \\
\hline Griechenland & 2,59 & 3,16 & 3 & 5,3 & 300 & 36 & 0 \\
\hline Guinea-Bissau & 1,89 & 2,97 & 0 & 3,8 & 100 & 6 & 0 \\
\hline Honduras & 2,3 & 2,46 & 0 & 7,1 & 128 & 13 & 0 \\
\hline Israel & 6,77 & 7,37 & 2 & 120 & 120 & 62 & 0 \\
\hline Italien & 3,07 & 3,82 & 4 & 630 & 630 & 16 & 0 \\
\hline Kapverden & 2,05 & 2,14 & 0 & 4,5 & 72 & 19 & 0 \\
\hline Kolumbien & 4,95 & 5,96 & 0 & 5 & 166 & 37 & 1 \\
\hline Kroatien & 3,07 & 4,23 & 0 & 12,8 & 153 & 18 & 0 \\
\hline Lettland & 3,93 & 4,59 & 5 & 20 & 100 & 17 & 1 \\
\hline Luxemburg & 3,63 & 4,25 & 0 & 15 & 60 & 80 & 0 \\
\hline Malta & 2 & 2,08 & 0 & 5 & 69 & 35 & 0 \\
\hline Mazedonien & 2,76 & 3,2 & 0 & 20 & 120 & 19 & 0 \\
\hline Mexiko* & 3,68 & 3,77 & 2 & 40 & 500 & 71 & 0 \\
\hline Moldawien & 3,23 & 3,68 & 4 & 101 & 101 & 16 & 1 \\
\hline Mongolei & 2,05 & 2,26 & 5 & 2,9 & 76 & 6 & 0 \\
\hline Neuseeland & 2,78 & 3,07 & 5 & 120 & 120 & 80 & 0 \\
\hline Niederlande & 6,74 & 6,97 & 0,67 & 8,3 & 150 & 80 & 1 \\
\hline Nordzypern & 2,68 & 3,33 & 5 & 10 & 50 & 17 & 0 \\
\hline
\end{tabular}


Thomas Krumm

\begin{tabular}{|l|c|c|c|c|c|c|c|}
\hline & ENPP & ENEP & $\begin{array}{c}\text { Höhe der } \\
\text { Sperrklausel }\end{array}$ & $\begin{array}{c}\text { MW Wahl- } \\
\text { kreis }\end{array}$ & $\begin{array}{c}\text { Parlaments- } \\
\text { sitze }\end{array}$ & $\begin{array}{c}\text { Alter des } \\
\text { Parteien- } \\
\text { systems }\end{array}$ & $\begin{array}{c}\text { Wahl- } \\
\text { jahr }\end{array}$ \\
\hline Norwegen & 4,07 & 4,55 & 0 & 8,9 & 169 & 80 & 0 \\
\hline Österreich & 4,24 & 4,79 & 4 & 20,3 & 183 & 56 & 0 \\
\hline Paraguay & 3,43 & 4,36 & 0 & 4,4 & 80 & 21 & 0 \\
\hline Peru & 3,78 & 7,31 & 4 & 4,8 & 120 & 30 & 0 \\
\hline Polen & 2,82 & 3,32 & 5 & 11,2 & 460 & 20 & 0 \\
\hline Portugal & 3,13 & 3,83 & 0 & 10,5 & 230 & 34 & 0 \\
\hline Rumänien & 3,6 & 3,93 & 5 & 7,9 & 334 & 18 & 0 \\
\hline Russland & 1,92 & 2,22 & 7 & 450 & 450 & 17 & 0 \\
\hline Schweden & 4,51 & 4,78 & 4 & 11,6 & 349 & 80 & 1 \\
\hline Schweiz & 4,97 & 5,61 & 0 & 7,7 & 200 & 80 & 0 \\
\hline Serbien & 3,48 & 3,73 & 5 & 250 & 250 & 8 & 0 \\
\hline Slowakei & 4,01 & 5,53 & 5 & 150 & 150 & 18 & 1 \\
\hline Slowenien & 4,23 & 4,94 & 4 & 11 & 90 & 19 & 0 \\
\hline $\begin{array}{l}\text { Tschechische } \\
\text { Republik }\end{array}$ & 4,51 & 6,75 & 5 & 14,3 & 200 & 20 & 1 \\
\hline Türkei & 2,25 & 3,47 & 10 & 6,5 & 550 & 26 & 0 \\
\hline Ukraine & 3,3 & 4,17 & 3 & 450 & 450 & 19 & 0 \\
\hline Ungarn* & 2,82 & 2,86 & 5 & 152 & 386 & 20 & 1 \\
\hline Uruguay & 2,65 & 2,75 & 0 & 5,2 & 99 & 26 & 0 \\
\hline Zypern & 3,9 & 4,29 & 1,8 & 13,3 & 56 & 22 & 0 \\
\hline
\end{tabular}

Quelle: Gallagher 2013, Keefer 2012 und eigene Ergänzung.

Anmerkungen: * Werte nur für Listenstimmen; MW: Mittelwert; Wahlkreis nach Keefer 2012 (Code Mean District Magnitude House) und eigene Ergänzung.

nächst nur die Effekte der Sperrklauselhöhe und der durchschnittlichen Wahlkreisgröße geschätzt. In Modell 2 wird zusätzlich die Gesamtzahl der Parlamentssitze im Jahr 2010 berücksichtigt. Modell 3 nimmt das demokratische Alter des Parteiensystems auf (begrenzt auf maximal 80 Jahre). Modell 4 nimmt nur die beiden ,Zeitvariablen'Alter des Parteiensystems und die Dummy-Variable Wahljahr auf. Modell 5 enthält alle Variablen. 
Wie wirksam sperren Sperrklauseln?

Tabelle 5: Effekte auf die effektive parlamentarische Parteienzahl (ENPP) 2010

\begin{tabular}{|c|c|c|c|c|c|}
\hline & Modell 1 & Modell 2 & Modell 3 & Modell 4 & Modell 5 \\
\hline Sperrklauselhöhe & $\begin{array}{l}-, 020 \\
(, 105)\end{array}$ & $\begin{array}{l}-, 063 \\
(, 111)\end{array}$ & $\begin{array}{l}, 039 \\
(, 108)\end{array}$ & & $\begin{array}{l}, 010 \\
(, 100)\end{array}$ \\
\hline Wahlkreisgröße (log) & $\begin{array}{l}, 074 \\
(, 174)\end{array}$ & $\begin{array}{l}-, 010 \\
(, 188)\end{array}$ & $\begin{array}{l}-, 033 \\
(, 174)\end{array}$ & & $\begin{array}{l}-, 045 \\
(, 160)\end{array}$ \\
\hline Sitze Parlament & & $\begin{array}{l}, 002 \\
(, 002)\end{array}$ & $\begin{array}{l}, 001 \\
(, 002)\end{array}$ & & $\begin{array}{l}, 001 \\
(, 002)\end{array}$ \\
\hline $\begin{array}{l}\text { Alter Parteiensystem } \\
\text { (max. } 80 \text { Jahre) }\end{array}$ & & & $\begin{array}{l}(, 027 * * \\
(, 009)\end{array}$ & $\begin{array}{l}, 027 * * \\
(, 008)\end{array}$ & $\begin{array}{l}, 027 * * \\
(, 008)\end{array}$ \\
\hline $\begin{array}{l}\text { Wahljahr } \\
(0=\text { Nein, } 1=\mathrm{Ja})\end{array}$ & & & & $\begin{array}{l}1,428^{* *} \\
(, 474)\end{array}$ & $\begin{array}{l}1,412 * * \\
(, 488)\end{array}$ \\
\hline Konstante & $\begin{array}{l}3,484 \\
(, 531)\end{array}$ & $\begin{array}{l}3,400 \\
(, 534)\end{array}$ & $\begin{array}{l}2,452 \\
(, 590)\end{array}$ & $\begin{array}{l}2,420 \\
(, 348)\end{array}$ & $\begin{array}{l}2,229 \\
(, 548)\end{array}$ \\
\hline $\mathrm{R}^{2}$ & ,004 & 034 & ,200 &, 320 & ,388 \\
\hline korr. $\mathrm{R}^{2}$ &,- 042 &,- 034 & 122 & 289 &, 255 \\
\hline
\end{tabular}

Quelle: Eigene Berechnung nach Tab. 4. $\mathrm{N}=46$, Standardfehler in Klammern. ${ }^{*} \mathrm{p}<0,05 ;{ }^{*} \mathrm{p}<0,01$.

Wie aus Tabelle 5 hervorgeht, erreicht die Sperrklauselhöhe in keinem der getesteten Modelle ein Signifikanzniveau. Gleiches gilt für die durchschnittliche Wahlkreisgröße. Beide bleiben sogar weit entfernt von einem Signifikanzniveau. Das gleiche gilt auch für die dritte getestete Variable, die Gesamtzahl der Parlamentssitze. Auch eine geringfügig veränderte Fallauswahl bringt keine Veränderung dieser Ergebnisse. Eine deutliche Verbesserung der Ergebnisse kann erst mit der Aufnahme der beiden letzten Variablen erreicht werden. Bereits mit der Aufnahme des Alters des Parteiensystems kann der Anteil der aufgeklärten Varianz deutlich verbessert werden. Die höchste (angepasste) Varianzaufklärung mit knapp 29 Prozent wird in Modell 4 erreicht, das lediglich die beiden signifikanten ,Zeitvariablen“ aufnimmt. Im letzten Modell mit allen Variablen können die Prädiktoren immerhin noch 25 Prozent der Varianz der parlamentarischen Fragmentierung aufklären. Im Ergebnis haben das Alter eines Parteiensystems und die Wahljahrvariable (2010) die deutlichsten Einflüsse auf die Fragmentierung des Parteiensystems auf parlamentarischer Ebene.

Die Signifikanz der Wahljahr-Variable ist ein Hinweis darauf, dass Wahlen bzw. Wahlkämpfe zur Fragmentierung eines Parteiensystems beitragen können. In einem Wahljahr werden die Fragmentierungswerte , aktualisiert ${ }^{\star}$ und bringen somit die 
Entwicklungen der letzten Vorwahljahre zum Ausdruck. Allerdings bildet die Variablen nur einen kurzen Zeitraum vor bzw. bis $2010 \mathrm{ab}$. Für eine Verallgemeinerung auf eine kontinuierliche generelle Zunahme der Fragmentierung von Wahl zu Wahl müssten Längsschnittdaten getestet werden. In Verbindung mit der Altersvariable kann das Ergebnis jedoch als ein Hinweis in diese Richtung interpretiert werden.

In Tabelle 6 werden die Modelle für die effektive Zahl elektoraler Parteien (ENEP) getestet. Auch auf Ebene der effektiven Zahl der zur Wahl angetretenen Parteien gibt es keinen Zusammenhang mit den ersten drei Variablen, dafür aber wieder mit den beiden Zeitvariablen. Allerdings wird nun für das Alter des Parteiensystems nur noch eine Irrtumswahrscheinlichkeit auf dem Niveau von fünf Prozent erreicht, während für den Wahljahr-Dummy wieder das Ein-Prozent-Niveau erreicht wird.

Tabelle 6: Effekte auf die effektive elektorale Parteienzahl (ENEP) 2010

\begin{tabular}{|c|c|c|c|c|c|}
\hline & Modell 1 & Modell 2 & Modell 3 & Modell 4 & Modell 5 \\
\hline Sperrklauselhöhe & $\begin{array}{l}, 046 \\
(, 116)\end{array}$ & $\begin{array}{l}, 008 \\
(, 124)\end{array}$ & $\begin{array}{l}, 100 \\
(, 125)\end{array}$ & & $\begin{array}{l}, 068 \\
(, 116)\end{array}$ \\
\hline Wahlkreisgröße (log) & $\begin{array}{l}, 002 \\
(, 192)\end{array}$ & $\begin{array}{l}-, 071 \\
(, 209)\end{array}$ & $\begin{array}{l}-, 091 \\
(, 200)\end{array}$ & & $\begin{array}{l}-, 105 \\
(, 186)\end{array}$ \\
\hline Sitze Parlament & & $\begin{array}{l}, 002 \\
(, 002)\end{array}$ & $\begin{array}{l}, 001 \\
(, 002)\end{array}$ & & $\begin{array}{l}, 001 \\
(, 002)\end{array}$ \\
\hline $\begin{array}{l}\text { Alter des Parteiensys- } \\
\text { tems (max. } 80 \text { Jahre) }\end{array}$ & & & $\begin{array}{l}, 024^{*} \\
(, 011)\end{array}$ & $\begin{array}{l}, 023 * \\
(, 009)\end{array}$ & $\begin{array}{l}, 024^{*} \\
(, 010)\end{array}$ \\
\hline $\begin{array}{l}\text { Wahljahr } \\
(0=\text { Nein, } 1=\mathrm{Ja})\end{array}$ & & & & $\begin{array}{l}1,604 * * \\
(, 551)\end{array}$ & $\begin{array}{l}1,567^{* *} \\
(, 566)\end{array}$ \\
\hline Konstante & $\begin{array}{l}4,178 \\
(, 586)\end{array}$ & $\begin{array}{l}4,105 \\
(, 594)\end{array}$ & $\begin{array}{l}3,258 \\
(, 679)\end{array}$ & $\begin{array}{l}3,197 \\
(, 405)\end{array}$ & $\begin{array}{l}3,010 \\
(, 636)\end{array}$ \\
\hline $\mathrm{R}^{2}$ & ,004 & 023 & ,131 & ,248 & ,271 \\
\hline korr. $\mathrm{R}^{2}$ &,- 042 &,- 047 & 047 & ,213 & , 180 \\
\hline
\end{tabular}

Quelle: Daten nach Tabelle 4; $\mathrm{N}=46$, Standardfehler in Klammern. ${ }^{*} \mathrm{p}<0,05 ; * * \mathrm{p}<0,01$.

Als Ergebnis der multiplen Regression von 46 Verhältniswahlsystemen ist festzuhalten, dass von den fünf getesteten Variablen (Sperrklauselhöhe, durchschnittliche Wahlkreisgröße, Parlamentsgröße, Alter des Parteiensystems und Wahljahr-Dummy) für die Höhe der Sperrklausel auf nationaler Ebene wie auch für die durchschnittliche Wahlkreisgröße und die Parlamentsgröße kein signifikanter Einfluss auf die parlamentarische und elektorale Fragmentierung der Parteien festgestellt werden kann. 
Demgegenüber brachten die Variablen Alter des Parteiensystems und Wahljahr eine deutliche Verbesserung der Modellanpassung. Dieser Aspekt wäre in einer Längsschnitt-Untersuchung weiter zu vertiefen. Möglicherweise tragen (häufigere) Wahlen zu einer weiteren Fragmentierung des Parteiensystems bei, worauf beispielsweise die höhere Signifikanz der Wahljahr-Variable auf elektoraler Ebene (ENEP) hinweist. Bei der Interpretation dieser Variable ist auch zu berücksichtigen, dass möglicherweise in den Modellen nicht kontrollierte Drittvariablen den Effekt mit verursacht haben könnten. Ferner ist zu berücksichtigen, dass die Fragmentierungswerte nur in Wahljahren aktualisiert werden. Dadurch kann eine möglicherweise kontinuierliche Zunahme parteipolitischer Fragmentierung nur diskontinuierlich (im Wahlrhythmus) gemessen werden. Das signifikante Ergebnis für die Altersvariable zeigt dagegen an, dass eine mit der Zeit steigende Parteienfragmentierung auch ein Preis des Alters demokratischer politischer Systeme (mit Verhältniswahlsystem) sein kann. Ein signifikanter Effekt der Sperrklauselhöhe auf die parlamentarische und elektorale Fragmentierung konnte dagegen im vorliegenden Sample nicht festgestellt werden.

\section{Fazit: Wie wirksam verhindern Sperrklauseln parlamentarische Fragmen- tierung?}

Wie die staatliche Parteienfinanzierung sind Wahlrechtsfragen ein Feld, in dem die Parteien „,in eigener Sache“ aktiv werden (müssen). Sie sind zugleich „rule-maker“ und „rule-taker" und regeln als Akteure ihre unmittelbare institutionelle Umwelt (Jackson 2010: 64). Dadurch kann der Eindruck verstärkt werden, dass sie auch zu ihrem eigenen Vorteil aktiv werden. Nach Ansicht des Bundesverfassungsgerichts liegt gerade bei der Wahlgesetzgebung die Vermutung nahe, „dass die jeweilige Parlamentsmehrheit sich statt von gemeinwohlbezogenen Erwägungen vom Ziel des eigenen Machterhalts leiten lässt" (zit. nach Hipp 2013). Aus historischer Perspektive hat dies bereits Jesse für die Reform des Wahlgesetzes im Jahr 1953 beobachtet, als die Anwendung der Fünf-Prozent-Hürde auf die Ebene des gesamten Bundesgebietes angehoben wurde: „So konsequent eine sich auf das gesamte Bundesgebiet erstreckende Fünfprozentklausel auch war, so peinlich nahmen sich die taktischen Finessen der Parteien hinsichtlich der Erschwerung und Erleichterung der Sperrklausel aus“"(1985: 225). Auch bei der Einführung der Drei-Prozent-Hürde 
im Europawahlgesetz im Juni 2013 wurden ähnliche taktische Züge beobachtet. ${ }^{28}$ Daher liegt es nahe, Sperrklauseln aus theoretischer Perspektive auch unter dem Aspekt des Konkurrenzschutzes bzw. der Kartellparteien zu untersuchen.

Bei der Bundestagswahl 2013 ist die parlamentarische Parteienzahl erstmals seit den 1950er Jahren wieder gesunken, allerdings hat sich die elektorale Parteienzahl wie auch der Stimmenanteil für die nicht im Parlament vertretenen Parteien (15,72 \%) deutlich erhöht. Im Längsschnitt (Tab. 1) lassen sich nach dem Ausmaß der unwirksam gebliebenen Stimmen drei Perioden unterscheiden, wobei in der mittleren Periode (1972-1987) die unwirksamen Stimmen mit durchschnittlich 1,13 Prozent am niedrigsten geblieben sind.

Um auch den psychologischen Effekt einer Absenkung der Sperrklausel auf das Wahlverhalten zu berücksichtigen, wurden für die Bundestagswahlen seit 1957 drei Simulationen berechnet (Tab. 3). In allen drei Szenarien erfolgte der geringste durchschnittliche Anstieg der Parteienzahl beim Absenken der Sperrklausel von vier auf drei Prozent und der größte Anstieg beim Übergang von zwei auf ein Prozent. Für die Europawahlen seit 1994 (Tab. 2, nur unverändertes Wahlverhalten) ist der geringste Anstieg beim Übergang von drei auf zwei Prozent ermittelt worden. Für die Bundestagswahlen lässt dies eine Empfehlung für eine Drei-Prozent-Hürde bedenkenswert erscheinen, die einerseits die Wahlrechtsgleichheit weniger stark beeinträchtigt und zugleich effektiv einem starken Anstieg der Parteienzahl im Bereich unter drei Prozent vorbeugt. Auch wäre nach allen drei Simulationen lediglich einmal (1969) eine rechtsextreme Partei (NPD) bei einer Drei-Prozent-Hürde in den Bundestag eingezogen.

Im internationalen Vergleich fällt auf, dass Deutschland trotz einer hohen Sperrklausel noch relativ hohe Fragmentierungswerte aufweist. Dies wirft die Frage der generellen Geeignetheit des Instruments zur Verhinderung einer höheren Parteienfragmentierung auf. Von den an 46 Fällen mit Verhältniswahlrecht getesteten fünf Variablen konnte ein signifikanter Einfluss auf die parlamentarische und elektorale Fragmentierung des Parteiensystems lediglich für die Variablen Alter des Parteiensystems und Wahljahr festgestellt werden. Ein signifikanter Zusammenhang von Sperrklauselhöhe und effektiver Parteienzahl (ENEP, ENPP) ist in der vorliegenden Fallauswahl $(\mathrm{N}=46)$ nicht nachweisbar (Tab. 5 und 6). Dabei muss allerdings eingeschränkt werden, dass soziale Heterogenität sowie Grundmandatsklauseln und Sonderregelungen für Parteienbündnisse nicht kontrolliert wurden. Bei einer mit 46

28 „In großer Eile, in nur neun Tagen, peitschte der Bundestag im Juni das Gesetz durch. Fristen wurden verkürzt, zweite und dritte Lesung fielen auf denselben Abend, gegen Mitternacht. Da lagen den Abgeordneten noch nicht einmal die Protokolle der Sachverständigen-Anhörung vor, die kurz zuvor durchgeführt worden war." (Hipp 2013). 
Ländern immer noch relativ kleinen Fallauswahl ist auch nicht auszuschließen, dass sich die Ergebnisse bei einer veränderten bzw. größeren Fallauswahl noch verändern können. Auch steht das Ergebnis der multiplen Regression nicht im Widerspruch zu dem aufgezeigten Anstieg der Parteienzahl im Deutschen Bundestag (Tab. 3), die lediglich einen bivariaten Zusammenhang in einem konkreten Fall untersucht hat. Dies kommt allerdings durchaus der Entscheidung des Bundesverfassungsgerichts vom November 2011 entgegen, nach der allgemeine theoretische Begründungen der (Un-)Geeignetheit von Sperrklauseln nicht ausreichend sind, um Einschränkungen der Wahlrechtsgleichheit und der Chancengleichheit politischer Parteien zu rechtfertigen. Vielmehr muss die Begründung der Geeignetheit und Erforderlichkeit von Sperrklauseln immer am konkreten Fall erfolgen.

Für eine weitere theoretische Analyse und eine Bestimmung der „optimalen“ Höhe von Sperrklauseln bietet sich auch ein institutionenökonomisches Instrumentarium an (z. B. Buchanan/Tullock 2004). So haben etwa aus Sicht der Kartellparteienthese Sperrklauseln die Aufgabe, bestehende Kartelle zu schützen und eine Oligopol- bzw. „Kartellrente“ in Form zusätzlicher, verbilligter Stimmen zu erzielen. Aus dieser theoretischen Perspektive können die Kosten der Defragmentierung durch Sperrklauseln vergleichsweise einfach auf Kleinparteien externalisiert werden. Allerdings ergibt sich aus der Perspektive des Konkurrenzschutzes bzw. der Kartellparteienthese auch eine Umkehrung der Fragestellung. Erklärungsbedürftig ist dann nicht mehr nur der Anteil von Ländern mit (hohen) Sperrklauseln, sondern auch der immer noch relativ großer Länderanteil ohne Sperrklauseln. Des Weiteren wäre es auch interessant, die Auswirkung der durch Sperrklauseln mitbedingten parlamentarischen Parteienzahl auf die Koalitionsbildung und die Regierungsstabilität zu untersuchen. Wie das Beispiel der Bundestagswahl 2013 illustriert, muss eine niedrige parlamentarische Parteienzahl nicht automatisch die Koalitionsbildung erleichtern. Eine geringfügig niedrigere Sperrklausel hätte in diesem Fall die Optionen für die Regierungsbildung deutlich erhöht. 


\section{Literatur}

Amorim Neto, Octavio/Cox, Gary W., 1997: Electoral Institutions, Cleavage Structures, and the Number of Parties, in: American Journal of Political Science 41 (1), 149-174.

Anckar, Carsten, 1997: Determinants of Disproportionality and Wasted Votes, in: Electoral Studies 16 (4), 501-515.

Arndt, Felix, 2011: Aktueller Begriff: Sperrklauseln im europäischen Wahlrechtsverbund, Wissenschaftliche Dienste des Deutschen Bundestags, Berlin, http:// www.bundestag.de/dokumente/analysen/kategorien/institutionen1/index.html (Stand: 21.3.2014).

Becht, Ernst, 1990: Die 5 \%-Klausel im Wahlrecht. Garant für ein funktionierendes parlamentarisches Regierungssystem?, Stuttgart.

Behnke, Joachim, 2009: Überhangmandate bei der Bundestagswahl 2009. Eine Schätzung mit Simulationen, in: Zeitschrift für Parlamentsfragen 40 (3), 620-636.

Bischoff, Carina S., 2009: National level electoral thresholds: Problems and solutions, in: Electoral Studies 28 (2), 232-239.

Buchanan, James M./Tullock, Gordon, 2004: The calculus of consent. Logical foundations of constitutional democracy, Ann Arbor (zuerst 1962).

Clark, William R./Golder, Matt, 2006: Rehabilitating Duverger's Theory. Testing the Mechanical and Strategic Modifying Effects of Electoral Laws, in: Comparative Political Studies 39 (6), 679-708.

Cox, Gary W., 2005: Electoral Institutions and Political Competition, in: Claude Menard/Mary M. Shirley (Eds.), Handbook of New Institutional Economics, Dordrecht, 69-89.

Downs, Anthony, 1968: Ökonomische Theorie der Demokratie, Tübingen.

Falter, Jürgen W./Schoen, Harald (Hrsg.), 2005: Handbuch Wahlforschung, Wiesbaden.

Gallagher, Michael, 2013: Election indices dataset, www.tcd.ie/Political_Science/ staff/michael_gallagher/ElSystems/index.php (Stand: 18.10.2013).

Georgii, Harald, 2010: Gegenüberstellung der Landeswahlgesetze. Infobrief WD 3 - 3010 - 428/10, Deutscher Bundestag, Berlin.

Geys, Benny, 2006: District Magnitude, Social Heterogenity and Local Party System Fragmentation, in: Party Politics 12 (2), 281-297.

Helms, Ludger, 2001: Die Kartellparteien-These und ihre Kritiker, in: Politische Vierteljahresschrift 42 (4), 698-708.

Hipp, Dietmar, 2013: Europa: Hürde um Hürde, Der Spiegel 42/2013, 34. 
Jackson, Gregory, 2010: Actors and Institutions, in: Glenn Morgan et al. (Eds.), The Oxford Handbook of Comparative Institutional Analysis, Oxford, 63-86.

Jesse, Eckhard, 1985: Wahlrecht zwischen Kontinuität und Reform. Eine Analyse der Wahlsystemdiskussion und der Wahlrechtsänderungen in der Bundesrepublik Deutschland 1949 - 1983, Düsseldorf.

Jesse, Eckhard, 2009: Verhältniswahl und Gerechtigkeit, in: Gerd Strohmeier (Hrsg.), Wahlsystemreform. Sonderband 2009 der Zeitschrift für Politikwissenschaft, Baden-Baden, 105-131.

Katz, Richard S., 2011: Political Parties, in: Daniele Caramani (Ed.), Comparative Politics. Oxford, 219-236.

Katz, Richard S./Mair, Peter, 1995: Changing Models of Party Organization and Party Democracy. The Emergence of the Cartel Party, in: Party Politics 1, 5-28. Keefer, Philip E, 2012: Database of Political Institutions (DPI) 2012, http:// go.worldbank.org/2EAGGLRZ40 (Stand: 28.10.2013).

Klingemann, Hans-Dieter/Wessels, Bernhard, 2001: The Political Consequences of Germany's Mixed-Member System: Personalization at the Grass Roots?, in: Matthew Soberg Shugart/Martin P. Wattenberg (Eds.), Mixed-Member Electoral Systems. The Best of Both Worlds?, Oxford, 279-296.

Kramer, Urs/Bahr, Vanessa, 2012: Die verschiedenen Sperrklauseln im Wahlrecht auf dem Prüfstand, in: Zeitschrift für das Juristische Studium 2/2012, 184-194. Krumm, Thomas, 2013: Das politische System der Schweiz, München.

Laakso, Markku/Taagepera, Rein, 1979: Effective Number of Parties: A Measure with Application to West Europe, in: Comparative Political Studies 12, 3-27.

Lijphart, Arend, 1994: Electoral Systems and Party Systems. A Study of 27 Democracies, 1945-1990, Oxford.

Lijphart, Arend, 1999: Patterns of Democracy. New Haven.

Michels, Robert, 1989: Zur Soziologie des Parteiwesens in der modernen Demokratie. Untersuchungen über die oligarchischen Tendenzen des Gruppenlebens, Stuttgart (zuerst 1911).

Nohlen, Dieter, 2009: Wahlrecht und Parteiensystem, Opladen.

Nohlen, Dieter/Stöver, Philip, 2010: Elections in Europe, in: Dieter Nohlen/Philip Stöver (Hrsg.), Elections in Europe, Baden-Baden, 69-124.

Riker, William H., 1962: The Theory of Political Coalitions, New Haven.

Saalfeld, Thomas, 2012: Die schleichende Revolutionierung des britischen Wahlsystems 1997-2011, in: Zeitschrift für Politik 59 (2), 197-216.

Scarrow, Susan E., 2001: Germany: The Mixed-Member System as a Political Compromise, in: Matthew Soberg Shugart/Martin P. Wattenberg (Eds.), MixedMember Electoral Systems. The Best of Both Worlds?, Oxford, 55-69. 
Stoll, Heather, 2008: Social Cleavages and the Number of Parties. How the Measure You Choose Affect the Answer You Get, in: Comparative Political Studies 41 (11), 1439-1465.

Strohmeier, Gerd, 2013: Kein perfektes Wahlsystem, aber ein guter Kompromiss unter schwierigen Rahmenbedingungen, in: Zeitschrift für Politik 60 (2), 144-161.

Taagepera, Rein, 2002: Nationwide threshold of representation, in: Electoral Studies 21 (3), 383-401.

Taagepera, Rein/Shugart, Matthew Soberg, 1989: Seats and Votes. The Effects and Determinants of Electoral Systems. New Haven.

Tsebelis, George, 2002: Veto Players. How Political Institutions Work, Princeton. Winkler, Jürgen R., 2012: Wahlrecht und Wahlsystem, in: Oskar W.Gabriel/Bettina Westle (Hrsg.), Wählerverhalten in der Demokratie, Baden-Baden, 135-148.

Korrespondenzanschrift:

PD Dr. Thomas Krumm

Philipps-Universität Marburg

Institut für Politikwissenschaft

Wilhelm-Röpke-Straße $6 \mathrm{G}$

35032 Marburg

E-Mail: krumm@staff.uni-marburg.de 